\title{
Valores democráticos para tiempos de crisis. Hallazgos de dos teorías
}

\author{
Democratic values for crisis times. Findings of two theories
}

\author{
José Eduardo Jorge \\ Facultad de Periodismo y Comunicación Social; \\ Universidad Nacional de La Plata (Argentina) \\ jjorge@perio.unlp.edu.ar
}

\section{Resumen}

Desde la perspectiva de la cultura política, el artículo examina una diversidad de índices de democracia a la luz de las teorías de los valores de autoexpresión de Inglehart y Welzel y de los valores culturales de Schwartz, a fin de indagar la naturaleza de las orientaciones culturales que subyacen en distintos modos de conceptualizar y medir la democracia. El trabajo estudia teórica y empíricamente los puntos de convergencia de ambas teorías, investigando la relación de sus indicadores culturales con los diversos índices en una muestra de 100 países. Se utilizan dos medidas de "democracia efectiva" propuestas en la literatura, así como las elaboradas por Freedom House, Banco Mundial, Bertelsmann Stiftung y el Centro de Investigación en Ciencias Sociales de Berlín (WZB) y Universidad de Zúrich (UZH). Las variables culturales son usadas para predecir cada uno de estos índices de democracia en 
modelos de regresión multivariados que incluyen además una batería de indicadores económicos, políticos y sociales. Las conclusiones apuntan a contribuir al debate normativo en un momento en el que crece la discusión pública y académica sobre una crisis o declive mundial de la democracia.

Palabras clave: valores democráticos; cultura política; medición de la democracia.

\section{Abstract}

Using the political culture approach, the article examines a diversity of democracy indices in the light of Inglehart's and Welzel's theory of self-expression values and Schwartz's theory of cultural values, in order to investigate the nature of cultural orientations that underline different ways of conceptualizing and measuring democracy. The work analyzes theoretically and empirically the areas of convergence of both theories, investigating the relationship of their cultural indicators with the various indices in a sample of 100 countries. It applies two measures of "effective democracy" proposed in the literature, as well as those developed by Freedom House, World Bank, Bertelsmann Stiftung and Berlin Social Science Center (WZB) and University of Zurich (UZH). The cultural variables are used to predict each of these democracy indices in multivariate regression models that also include a battery of economic, political, and social indicators. The conclusions aim to contribute to the normative debate in the moment in which public and academic discussions on a crisis or global decline of the democracy are growing.

Keywords: democratic values; political culture; measures of democracy.

En una reciente entrevista, el filósofo canadiense Charles Taylor decía que es posible vivir en una democracia como un alienígena -un "resident alien", fueron sus palabras-, ocupado en los asuntos personales e indiferente a la trama colectiva. Pero podría ocurrir algo que cambiara esa actitud. "Si siento que estoy en verdad enojado o contento de vivir en esta sociedad, que vivo con plenitud esa experiencia o que estoy privado de ella, esto es un síntoma de que he sido capturado por la ética de la democracia" (Rothman, 2016).

El motivo del diálogo era la aparente "crisis de la democracia", la "desilusión" y la "pérdida de $\mathrm{fe}$ " en el sistema que se ha difundido entre los ciudadanos de muchas democracias, y que hoy es objeto de inquietud y discusión en los medios y el mundo intelectual y académico. 
En los últimos 20 años, según un estudio que la prensa recogió con gravedad, las generaciones jóvenes de varios países de Europa y EE.UU. se han vuelto más "cínicas" sobre el valor de la democracia. Comparadas con sus mayores, creen menos en su propia capacidad de influir en las políticas de gobierno y son más proclives a apoyar "alternativas autoritarias" (Foa y Mounk, 2016).

"La democracia es teleológica; un esfuerzo colectivo con un objetivo noble: la inclusión", dice Taylor, cuya reflexión resuena con fuerza en el contexto latinoamericano. Los ciudadanos, argumenta, solían hallar satisfacción personal participando en política. Y el cinismo, la idea de que el proceso democrático carece de sentido, podría tornarse una profecía autocumplida.

A los 85 años, el autor de Fuentes del Yo (1989) recuerda otra época de crisis, en la que muchos pensadores veían con recelo que la gente buscara significado en la vida política. Alrededor de la Segunda Guerra Mundial, alarmados por los totalitarismos europeos y sus movimientos de masas, Joseph Schumpeter y otros pensaban que lo mejor era "ir a las urnas cada cuatro años y elegir un equipo de elite". Taylor defiende otra ética. "Participar en nuestro propio autogobierno es un modo más elevado de ser".

La literatura académica de los últimos años ha insistido en señalar como síntomas de "crisis" la desafección política de los ciudadanos, la desconfianza en las instituciones y la insatisfacción con su desempeño, la sangría de afiliados a los partidos, la inefectividad de la representación y el acrecentado poder de actores exentos de control electoral, como los bancos centrales y las instituciones transnacionales (Ercan y Gagnon, 2014),

Las sociedades han quedado "divididas entre los deseos de la gente y el gobierno de los expertos" (Luce, 2017), como evidenció el triunfo de la opción de dejar la Unión Europea en el referéndum británico de 2016. Los encuentros del Foro Mundial de Davos, dice este analista, reflejan hoy "una visión del mundo que es inherentemente aprensiva de la opinión pública". Para Davos, "la democracia nunca es una cura" y los gobiernos "deben escuchar más a las multinacionales".

Ha sido finalmente el ascenso de la extrema derecha, el éxito de candidatos y partidos que movilizan actitudes xenófobas, racistas, misóginas y nativistas, lo que ha despertado el temor de que aún las democracias maduras puedan "desconsolidarse".

La expansión mundial de las instituciones representativas se frenó tras el cambio de siglo. Salvo en Túnez, el entusiasmo por la Primavera Árabe terminó en frustración. No solo el número de democracias dejó de crecer. La "transitología" adquirió "el sabor de las cenizas" (Art, 2012) y el foco de investigación giró del cambio democrático a la resiliencia de los autoritarismos.

A partir de 2006, el índice de Derechos Políticos y Libertades Civiles de la organización Freedom House registra 11 años seguidos de declive de las libertades democráticas en el mundo. Para la fundación alemana Bertelsmann, cuyo índice de Transformación evalúa 129 
países en desarrollo, en la última década aumentó en este grupo el "conflicto social, étnico y religioso". En 2016, sólo seis estados tuvieron una nota "muy buena" en su gestión de gobierno (Bertelsmann Stiftung, 2016).

Una visión clara de los valores que sustentan y profundizan la democracia es de importancia crucial cuando ésta sufre fuertes presiones internas y externas. Al ayudar a esclarecer los valores que subyacen en nuestras actitudes y prácticas, el enfoque de la cultura política puede contribuir al debate normativo.

En este artículo parto de dos teorías convergentes, altamente formalizadas y con sólido respaldo empírico -las desarrolladas por Inglehart y Welzel y por Schwartz-, para explorar la naturaleza de las orientaciones culturales que surgen de analizar una diversidad de enfoques conceptuales y operativos de la democracia.

\section{La discusión sobre la "crisis de la democracia"}

Para producir el fenómeno del nacionalismo xenófobo han confluido dos tendencias de largo plazo, exacerbadas por efectos de coyuntura (Inglehart y Norris, 2017, 2016). Una es el aumento de la desigualdad a partir de los 70 -más agudo en EE.UU. y Gran Bretaña-, junto a la erosión del Estado de bienestar y al hecho de que en los últimos 20 años las clases medias de los países industrializados fueron las grandes perdedoras de la globalización (Piketty, 2014; Milanovic, 2016; Baldwin, 2016). El otro desarrollo es una reacción de los grupos con valores tradicionales arraigados contra el rápido cambio cultural ocurrido desde los 60 , que ha dado lugar incluso a las llamadas "guerras culturales".

El peso creciente de nuevos valores modificó gradualmente la agenda política y suscitó realineamientos partidarios (Inglehart, 1997, 1990). Se formó una "nueva izquierda" de sectores medios educados enfocada en cuestiones culturales, mientras parte de los trabajadores con menor instrucción, aferrados a la tradición y los valores familiares, migró hacia partidos de derecha. El acento en la "política de identidad", reemplazando a las demandas redistributivas de la centroizquierda de posguerra, no ayudó a corregir el avance de la inequidad.

Agravaron estas tendencias la crisis financiera de 2008, la relocalización de empresas en países con bajo costo de mano obra, la automatización y la inmigración masiva, que afectan los ingresos, las oportunidades y el sentido de identidad cultural de los grupos económicamente más inseguros: "left-behinds" británicos, "couches moyennes" franceses o "squeezed middle" estadounidense (Luce, 2017; Hochschild, 2016).

La posición de la democracia como el modelo global de legitimidad política y de vía hacia la prosperidad podría resentirse con la percepción de que sus expresiones maduras están en 
problemas, el giro del poder geopolítico a favor de Asia, el éxito de la estrategia de desarrollo china y el renovado vigor de los autoritarismos (Diamond y Plattner, 2015).

Diamond destaca que algunas democracias nuevas se han movido hacia el "autoritarismo competitivo" o a una "zona gris", debido a "degradaciones sutiles e incrementales de los derechos y procedimientos democráticos". La erosión "no es siempre evidente" y deriva del "mal gobierno", del deterioro del "estado de derecho" y la "transparencia", incluida la corrupción (Ibídem: 108-9). Al separar el componente que mide estos dos aspectos en el índice de Freedom House, comprueba que todas las regiones del mundo tienen peor puntaje en estado de derecho y transparencia que en derechos políticos o libertades civiles.

En contraste, examinando varios índices de democracia, Levitsky y Way (2015) concluyen que la democracia global "ha permanecido estable" en estos 10 años y "mejorado marcadamente" desde los 90. Hoy se percibe una recesión porque las aperturas políticas que produjo la caída del Muro de Berlín han sido interpretadas y evaluadas, a la luz de la exitosa ola de la década previa, como "transiciones democráticas", cuando eran "crisis sin precedentes del autoritarismo".

Estos análisis llaman la atención sobre los criterios teóricos y metodológicos de medición de la democracia (Jorge, 2010: 36-54). Con pocas excepciones, los índices se basan solo en el juicio de los expertos. Un temprano estudio de Bollen y Paxton (2000) descubrió "errores sistemáticos" causados por "los sesgos perceptuales de los jueces que hacen los rankings subjetivos". Coppedge y Gerring (2011) van más lejos al señalar que "no hay consenso sobre cómo conceptualizar y medir los regímenes políticos". El debate, dicen, envuelve a la vez lo que las democracias "son" y lo que "deberían ser".

Trabajos del Centro de Investigación en Ciencias Sociales de Berlín (WZB), que elabora el Barómetro de la Democracia con la Universidad de Zúrich, arrojan que la mayoría de los regímenes del mundo no son ni democracias plenas ni dictaduras, sino casos intermedios (Merkel, 2016). Los sistemas democráticos, para este autor, sufren crisis "parciales" pero no una "crisis integral" capaz de desconsolidarlos. Afrontan empero dos "desafíos dramáticos", la globalización y la desigualdad, que podrían llevarlos a una crisis si siguen sin encontrar soluciones adecuadas.

\section{La cultura política democrática}

La tesis central del enfoque de la cultura política es que la democracia -y cualquier régimen político estable- requiere una cultura compatible arraigada en la sociedad. Definimos la cultura política como el conjunto de valores, creencias y pautas de conducta prevalecientes en una sociedad que influyen en el proceso político (Jorge, 2010). 
Planteada hace 50 años por Almond y Verba (1963) y Eckstein (1966), la "hipótesis de la congruencia" ha ganado creciente aceptación entre los académicos y promotores de la democracia (Clark et al., 2012; Coppedge, 2012). Un sistema específico de orientaciones culturales parece ser esencial para la solidez, profundidad y efectividad del sistema.

La naturaleza exacta de este sistema ha sido motivo de debate (Inglehart, 1990 y 1997; Putnam, 1993 y 2000; Almond y Verba, 1989). La obra de Inglehart y Welzel Modernization, Cultural Change, and Democracy (2005) fue un hito que fijó los contornos de la discusión actual. Integrando un enorme volumen de datos, entre ellos los recopilados desde los 80 por World Values Survey (WVS) en 81 países, este "ambicioso libro", dice Coppedge (2012: 224), propone "una teoría de vasto alcance del cambio cultural y la democratización" y "presenta masiva evidencia y sofisticados análisis en apoyo a sus argumentos clave".

Su conclusión es que la probabilidad de que un país tenga una democracia en la que rijan efectivamente los derechos de los ciudadanos depende del grado en que está difundido en la sociedad un sistema de valores de "autoexpresión" o "emancipación". Este síndrome enfatiza la "libertad de elección", pero no de un modo egoísta, pues va unido a un acento en la "igualdad de oportunidades". Es una forma de "individualismo altruista", que orienta a las personas hacia el civismo y la justicia social. Uno de sus rasgos es la confianza generalizada en los demás (Jorge, 2016a). El ascenso de los valores de emancipación es resultado del desarrollo posindustrial -junto a la creación del Estado de bienestar-, que al reducir la inseguridad económica hace emerger en las personas necesidades de orden superior. Refleja los cambios generacionales de cultura, que operan como variable intermedia entre el cambio económico y el político-institucional. 


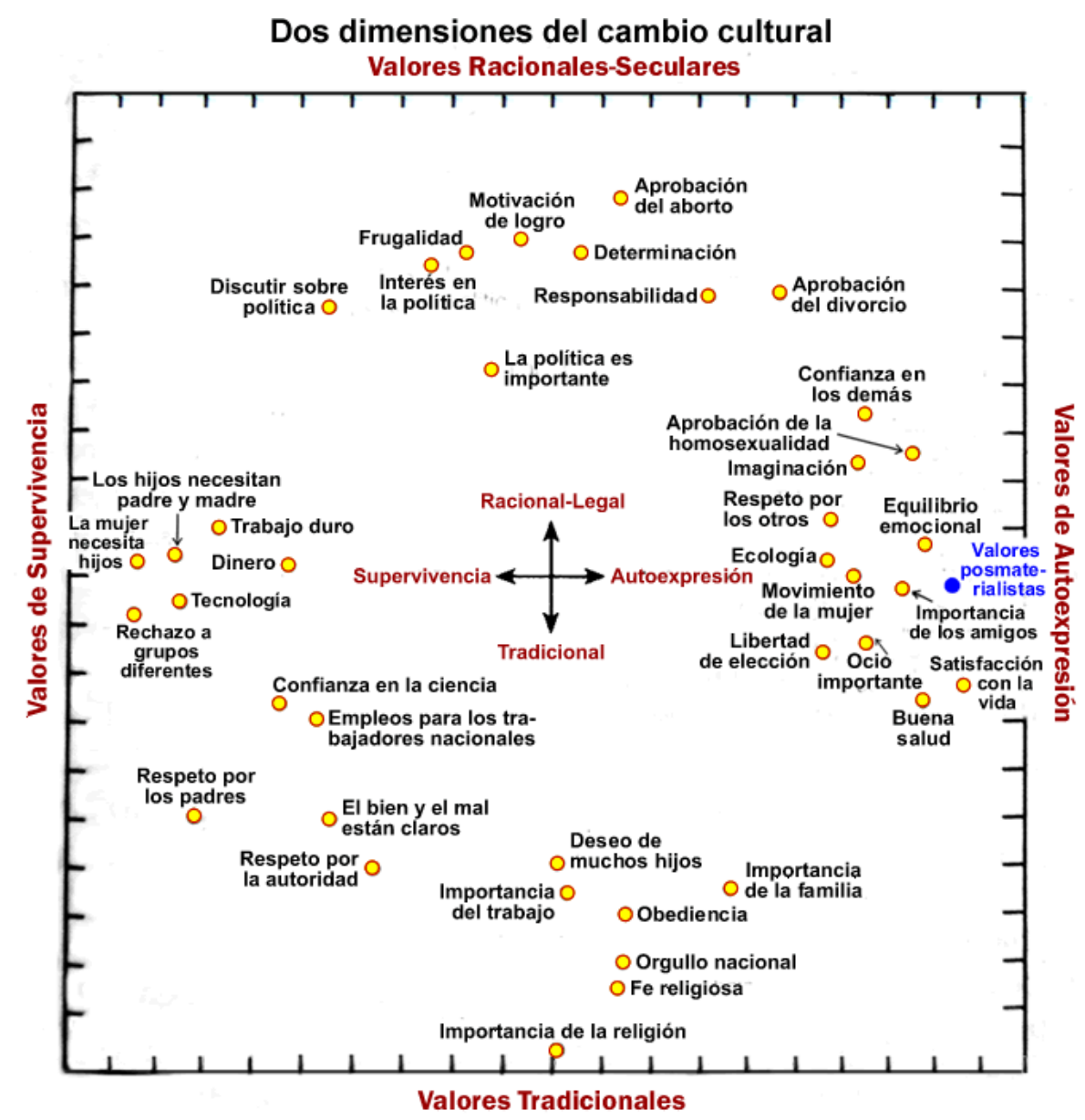

Fuente: Elaboración propia a partir de Inglehart (1997: 82)

Figura 1

La era industrial, al reemplazar los valores tradicionales por los racionales y seculares, produce la "secularización de la autoridad", que es compatible con la democracia, pero también con el fascismo, el socialismo de estado y otros regímenes autoritarios. La era posindustrial, con su giro desde los valores de "supervivencia" a los de autoexpresión, emancipa a los individuos de todas las formas de autoridad, una tendencia inherentemente prodemocrática. La Figura 1, surgida de un análisis factorial con datos de WVS en el nivel de los países, refleja las dos dimensiones en las que varía la prioridad que las sociedades asignan a valores fundamentales. El desarrollo avanzado también expande la capacidad humana de elegir generando un proceso masivo de "movilización cognoscitiva". Los entornos de trabajo complejos exigen el manejo de información, el pensamiento y la decisión independiente, creatividad y habilidades de comunicación y organización. La difusión de la educación superior y de los medios y tecnologías de comunicación acrecienta la autonomía intelectual e informativa de los individuos. 
En Freedom Rising (2013). Welzel amplió y refinó el edificio teórico. Su "lógica de base", dice allí, es que "las menguantes presiones existenciales abren la mente de las personas; las hacen priorizar la libertad sobre la seguridad, la autonomía sobre la autoridad, la diversidad sobre la uniformidad y la creatividad sobre la disciplina". Ese estado mental es también "la fuente de tolerancia y solidaridad más allá del propio grupo" (2013: xxiii).

Si la cultura política de una sociedad incluye una gran variedad de elementos, su núcleo consiste en una estructura de valores básicos. Aspectos como las normas y actitudes tendrían una posición más periférica.

Los valores son creencias cargadas de afecto; concepciones generales de los fines deseables e indeseables, que funcionan como "un poderoso regulador motivacional de la conducta humana" (Inglehart y Welzel, 2005: 23). Schwartz (2009) destaca que los valores predominantes son el rasgo más central de la cultura, pues impulsan la coherencia de sus diversas manifestaciones. Las normas, las prácticas cotidianas, la organización y las políticas de las instituciones, expresan los valores prevalecientes.

Esto es importante al evaluar el impacto de las actitudes de apoyo, confianza y satisfacción con la democracia y sus instituciones, que algunos -en la tradición de David Easton (1965)consideran fundamentales. Para Foa y Mounk (2016), que los jóvenes crean menos "esencial" que sus mayores vivir en democracia es un signo grave de deslegitimación del sistema.

Inglehart (2016a) admite un deterioro, más como un "efecto de coyuntura" concentrado en EE.UU. por el "masivo aumento" de la desigualdad y la "desproporcionada influencia de los billonarios" en el gobierno. Pero subraya que siguen aumentando los "indicadores clave" de apoyo, como la "tolerancia a la homosexualidad" y la "aceptación de la igualdad de género", que predicen los niveles de democracia "mucho mejor" que las meras opiniones favorables al sistema. Éstas suelen encubrir, entre quienes poseen otros valores, "interpretaciones autoritarias de la democracia" (Welzel y Kirsch, 2017), como la idea de que uno de sus rasgos "esenciales" es la "obediencia a los gobernantes".

Coppedge (2012: 224) destaca el "creciente consenso" de que los "ciudadanos críticos" que plantean desafíos a las elites (Dalton y Welzel, 2014), y un grado de desconfianza en las instituciones, son "saludables para la democracia". Para Diamond (2015:115), la mutación de valores que el cambio económico y tecnológico no cesa de promover, "erosionando todas las formas de autoridad y empoderando a los individuos", es el factor que tiende a minar la legitimidad de todos los regímenes autoritarios.

\section{Convergencia de dos teorías}


Algunas críticas recientes a la teoría de los valores de emancipación provienen de la perspectiva de la "democracia sin demócratas" (Dahlum y Knutsen, 2015; Hadenius y Teorell, 2005). Para Inglehart y Welzel (en adelante I \& W), la democracia estable y profunda no surge de los acuerdos de elite o de la ingeniería institucional. Los valores motivan a la gente a "presionar por libertad, derechos civiles y políticos efectivos y un gobierno que dé respuestas genuinas, y a ejercer continua vigilancia para asegurar que las elites gobernantes las sigan dando" (2005: 300).

Otros señalan áreas donde la teoría requeriría mayor desarrollo o precisión. Coppedge (2012: 226) nota la dificultad de lograr "validez conceptual" usando preguntas estandarizadas en diversos contextos culturales, pues los entrevistados pueden interpretarlas de modo sutilmente distinto. Una solución es el análisis factorial, que reduce una multiplicidad de indicadores a una - más dimensiones subyacentes. Pero la interpretación de estas dimensiones -una combinación de arte y ciencia- es disputable, más si se afirma haber descubierto "valores universales" (Ibídem: 227). Coppedge se pregunta si "autoexpresión" es un nombre adecuado y si envuelve realmente todos los indicadores incluidos.

Existe empero otra teoría de los valores ampliamente aceptada, desarrollada por el psicólogo Shalom H. Schwartz, que coincide en puntos esenciales con la de I \& W (Schwartz, 2009, 2008b, 2006, 1992; Dobewall y Rudnev, 2014). Esta última es inductiva, construida a partir de la evidencia empírica; la de Schwartz es hipotético-deductiva, basada en inferencias sobre las respuestas que deben dar las culturas y los individuos a problemas universales. Ambas usan instrumentos y muestras distintas para medir los valores.

En el nivel de la sociedad, Schwartz distingue siete orientaciones culturales de valor, que forman tres dimensiones bipolares. La Figura 2 es una proyección bidimensional del patrón de intercorrelaciones entre 47 valores en el nivel cultural (no individual). Surge de los datos de los cuestionarios de Schwartz Value Survey (SVS) administrados entre 1988 y 2005 a muestras de grupos de 72 países, principalmente maestros y estudiantes.

La descripción de cada valor en la figura sintetiza a la incluida en los cuestionarios de SVS. Las orientaciones adyacentes -por ejemplo, autonomía e igualitarismo, o inmersión y jerarquía- son compatibles (correlacionan positivamente) entre sí; las opuestas -autonomía e inmersión, o igualitarismo y jerarquía- están en conflicto. La cultura, en esta concepción, está "fuera" de los individuos y entraña las "presiones" -estímulos psicológicos y expectativas de rol- a las que aquéllos están expuestos (Jorge, 2017:180-83).

La dimensión autonomía versus inmersión en el grupo, que opone valores como "libertad", "amplitud mental", "creatividad" y "vida excitante" a los de "obediencia", "orden social" o "respeto por la tradición", se traslapa empíricamente en grado importante con la dimensión autoexpresión versus supervivencia de I \& W (Schwartz, 2009: 134). Ambas reflejan la medida 
en que la cultura estimula o no a los individuos a "expresar su carácter único e independencia de pensamiento, acciones y sentimientos".

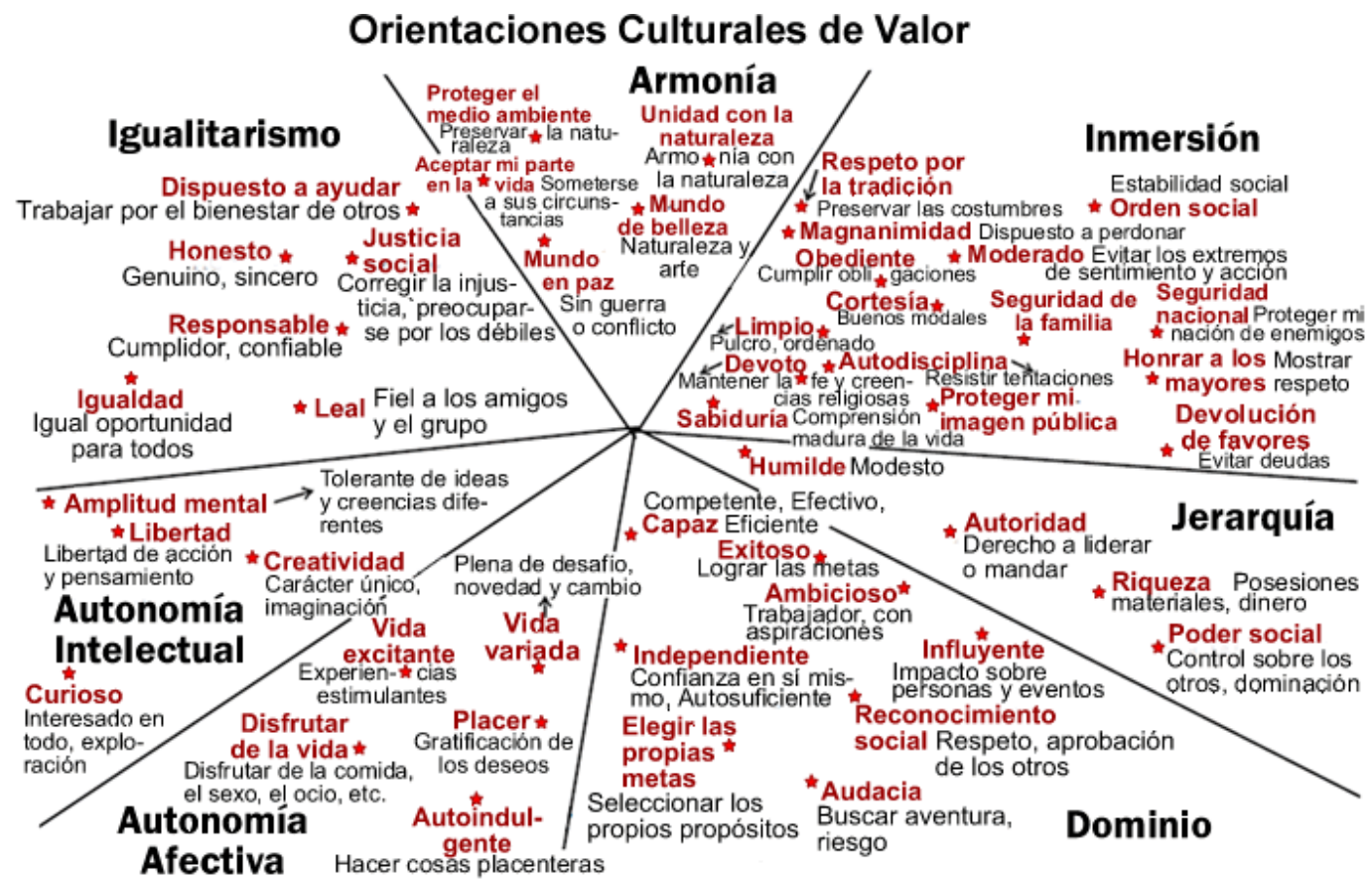

Fuente: Elaboración propia a partir de Schwartz (2009: 132)

Figura 2

Igualitarismo versus jerarquía -que opone, por ejemplo, "igual oportunidad para todos" y "justicia social" a "autoridad" y "riqueza"- también se superpone, aunque menos, con autoexpresión versus supervivencia. Las dos dimensiones envuelven la "igualdad entre grupos, tolerancia y confianza". Autonomía versus inmersión -y, en algún grado, armonía versus dominio- se traslapa además con valores tradicionales versus racionales y seculares.

Estas coincidencias entre teorías con enfoques conceptuales y métodos disímiles son muy notables y "apoyan con fuerza" la idea de que sus dimensiones "capturan aspectos reales y robustos de diferencia cultural" (Ibídem; Schwartz, 2006).

Los valores de autonomía individual e igualitarismo tienen una fuerte relación positiva con el grado de democracia de los países, el estado de derecho y la transparencia o corrupción percibida (Schwartz, 2006; Licht et al., 2007).

La Figura 3 muestra en dos dimensiones la posición de los grupos nacionales en la estructura de orientaciones. El "perfil" de los países refleja la coherencia de la estructura de orientaciones. China, por caso, es alta en jerarquía y en el adyacente dominio -que enfatiza el control, el cambio o la explotación del entorno natural y social, en lugar de armonizar con él, entenderlo y apreciarlo-. A la vez, es baja en igualitarismo y armonía. 
Del mapa emergen ocho "regiones culturales", con muchas coincidencias con las de I \& W (Figura 4). Lo que suele llamarse "cultura occidental" tiene importantes diferencias internas. Europa Occidental es más alta en igualitarismo, autonomía intelectual y armonía. Los países de habla inglesa enfatizan la autonomía afectiva y el dominio (Schwartz, 2009). EE.UU acentúa aún más el dominio y menos el igualitarismo, la autonomía intelectual y la armonía; su perfil es el de una cultura emprendedora y pragmática. La cultura de América Latina es próxima al promedio mundial en las 7 orientaciones. Argentina y Chile estimulan más la autonomía intelectual y el igualitarismo, Bolivia la inmersión y Perú la jerarquía.

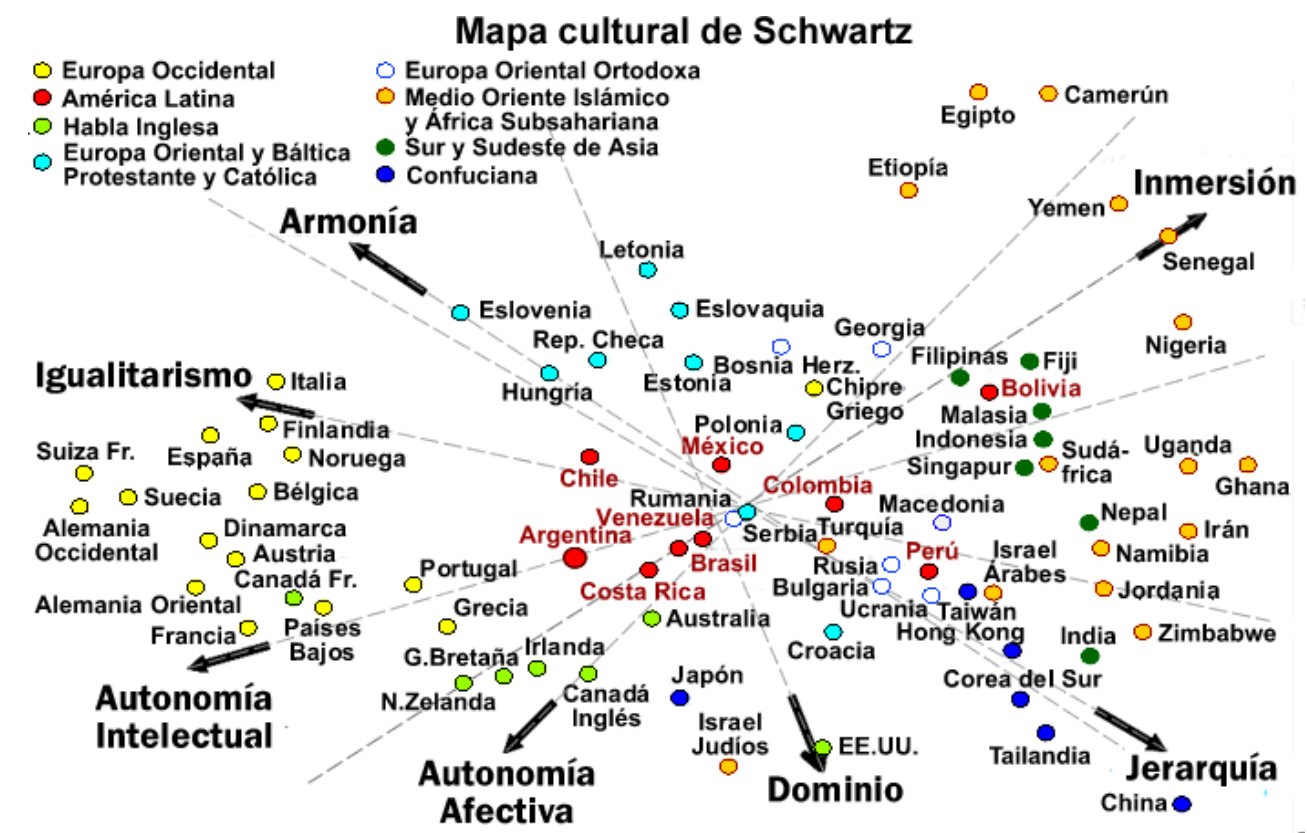

Fuente: Elaboración propia a partir de Schwartz (2009: 135)

Figura 3

Este análisis contradice la visión convencional del individualismo, ligado a la búsqueda egoísta del interés personal -visión presente en Hofstede (1980)-, y converge con el individualismo "altruista" de los valores de autoexpresión (Welzel, 2010). Para Schwartz (2009: 137), la cultura de Europa Occidental es "individualista" en el sentido de que enfatiza la autonomía intelectual y afectiva, y desalienta la jerarquía y la inmersión en el grupo. Pero al estimular el igualitarismo y la armonía -y desalentar el dominio- "llama a la preocupación desinteresada por el bienestar de los otros" y a amoldarse al mundo natural y social en vez de controlarlo.

Pese a haber sido hasta ahora poco utilizada en el estudio de la democratización, la teoría de Schwartz refuerza hipótesis fundamentales sobre la naturaleza de la cultura política democrática y abre nuevas vías de investigación.

La tesis de la congruencia no queda limitada al tipo de régimen. Las políticas de gobierno compatibles con los valores prevalecientes son vistas por la población como "legítimas" y 
"naturales"; las incompatibles, como ilegítimas. Los valores predominantes proveen justificación e influyen, por ejemplo, en políticas como la asignación del gasto público y la extensión de la red de seguridad social, que varían entre países con distinto perfil cultural (Schwartz, 2008b).

\section{Mapa cultural de Inglehart y Welzel}

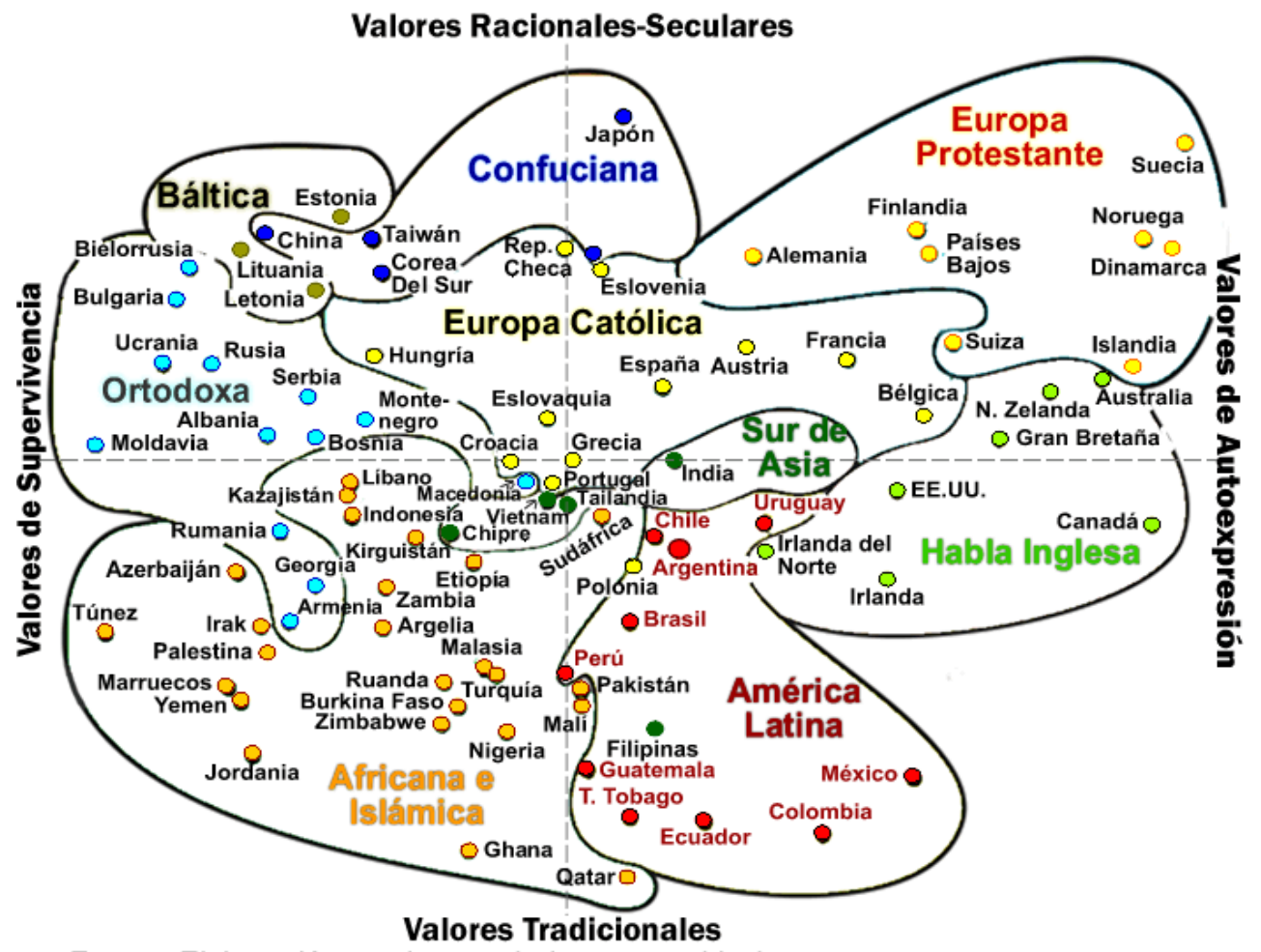

Fuente: Elaboración propia a partir de www.worldvaluessurvey.org

Figura 4

\section{Aplicación de las teorías a medidas distintas de democracia}

En Jorge (2015) presenté un índice de cultura política democrática (ICPD), que construí mediante una selección sistemática de valores de autoexpresión a partir de datos de WVS. Aplicando el análisis de regresión multivariado con una muestra de más de 80 países, mostré su capacidad de predecir el nivel de democracia medido por el índice de Freedom House. En Jorge (2017) usé el mismo enfoque para predecir el estado de derecho y la corrupción percibida. En ambos trabajos examiné puntos de convergencia entre las teorías de I \& W y Schwartz.

Aquí busco extender y profundizar el análisis con una multiplicidad de índices de democracia, el ICPD, un conjunto actualizado de datos de los valores culturales de Schwartz y una batería ampliada de variables de control económicas, políticas y sociales. 
Los datos son de dos periodos: 2003-2014 y 1990s-2007. Los de Schwartz (2008a), para 75 países, fueron recopilados por SVS entre 1988 y 2007, la mayor parte a mediados de los 90. Calculé el ICPD y sus componentes entre 1990 y 2007 para 66 de esos países. La Tabla 1 presenta sus correlaciones con los puntajes de los valores culturales de Schwartz.

\begin{tabular}{|c|c|c|c|c|c|c|c|c|}
\hline \multicolumn{9}{|c|}{ Correlaciones entre Cultura Politica Democrática y Valores Culturales de Schwartz 1990s-2007 } \\
\hline & $\begin{array}{c}\text { ICPD } \\
\text { Base }\end{array}$ & $\begin{array}{c}\text { ICPD Re- } \\
\text { finado }\end{array}$ & $\begin{array}{l}\text { Asp. de } \\
\text { Libertad }\end{array}$ & $\begin{array}{l}\text { Respeto } \\
\text { por Otros }\end{array}$ & $\begin{array}{l}\text { Igualdad } \\
\text { de Género }\end{array}$ & $\begin{array}{l}\text { Acción } \\
\text { Política }\end{array}$ & $\begin{array}{l}\text { Conf. No } \\
\text { Especif. }\end{array}$ & $\begin{array}{l}\text { Conf. Ge } \\
\text { neralizada }\end{array}$ \\
\hline Autonomía (1) & $0,80 \star \star \star *$ & $0,79 * \star \star$ & $0,70 * \pi *$ & $0,79 \approx \star \star x$ & 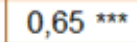 & 0,74 *** & 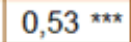 & 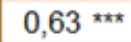 \\
\hline Autonomia Intelectual & $0,75^{\star \star \star *}$ & 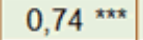 & $0,69 * \star * \star$ & $0,79^{\star \star \star \star}$ & 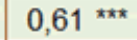 & $0,65^{\star \star \star}$ & $0,44 * \star x \star$ & 0,52 *** \\
\hline Autonomia Afectiva & 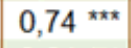 & $0,72 * \pi \star$ & 0,62 *** & $0,68^{\star \star *}$ & 0,60 **ะ & $0,71^{\star * \star}$ & $0,54^{\star * *}$ & 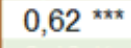 \\
\hline Igualitarismo & $0,64 * * \pi$ & $0,68^{\star \star \star}$ & $0,73^{\star * \star *}$ & $0,60^{* \times *}$ & 0,63 *ะส & 0,56 *** & $0,22 \nmid$ & 0,40 * \\
\hline Armonia & $0,40^{\star \star \star *}$ & $0,49^{\star \star \star \star}$ & $0,38^{\star \star}$ & $0,46^{* * *}$ & $0,32^{\star \star \star *}$ & 0,28 * & $0,16 \mathrm{~ns}$ & $0,28 \dagger$ \\
\hline Inmersión & $-0,82^{\star \star \star *}$ & $-0,80 * \star \star$ & $-0,75^{\star \times \star}$ & $-0,79$ *ะ* & 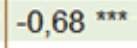 & $-0,76^{\star \star \star *}$ & $-0,49 * * \star$ & $-0,58$ *ะ* \\
\hline Jerarquia: & $-0,53^{* * *}$ & $-0,59^{* \times \mathrm{x}}$ & $-0,50^{* \times *}$ & $-0,60^{\mathrm{x} \times \mathrm{x}}$ & $-0,48^{* \times *}$ & $-0,46$ *ะ* & $-0,19 \mathrm{~ns}$ & $-0,44 \approx *$ \\
\hline Dominio & $-0,12 \mathrm{~ns}$ & $-0,25 \mathrm{~ns}$ & $-0,21+$ & $-0,21+$ & $-0,13$ ns & $-0,03 \mathrm{~ns}$ & $0,00 \mathrm{~ns}$ & $-0,20 \mathrm{~ns}$ \\
\hline Autonomia menos Inmersión ${ }^{(2)}$ & 0,82 *ะ* & $0,81 * \approx \times$ & $0,74 * \times \times$ & $0,81 \times 1 \times x$ & 0,68 *ะ* & $0,76^{\star \star \star}$ & $0,52^{* * *}$ & 0,62 *** \\
\hline Igualitarismo-menos Jerarquia ${ }^{(2)}$ & $0,64 * \pi \star$ & $0,69 * \star \star$ & $0,66^{\star * \star}$ & $0,68^{* * *}$ & 0,61 *ะः & $0,56^{\star * \star}$ & $0,23+$ & 0,48 *夫 \\
\hline Armonía menos Dominio ${ }^{(2)}$ & $0,34 \star \star \star *$ & 0,45 ** & $0,36^{\star *}$ & $0,43^{* * *}$ & 0,28 * & $0,22+$ & $0,12 \mathrm{~ns}$ & $0,28+$ \\
\hline $\mathrm{N}^{0}$ de Países & 66 & 40 & 64 & 65 & 59 & 65 & 66 & 41 \\
\hline
\end{tabular}

Correlaciones calculadas con los paises de la Tabla del anexo cono unidades de análisis. Los valores de los indices ICPD y sus componentes son del periodo 1990-2008. Los puntajes de los valores culturales de Schwartz están disponibles en Schwartz (2008a). La Tabla del anexo presenta los principales datos por pais. (1) Promedio de Autonomia Intelectual y Afectiva. (2) Puntaje que resulta de restar el de una orientación al de la orientación polar opuesta (Schwartz, 2006:161-68): ${ }^{* * *} p<0.001{ }^{* *} p<0.01{ }^{*} p<0.05 \nmid p<0.1$ ns: no significativo

Tabla 1

El ICPD Base es un promedio ponderado de las proporciones de la población de cada país que firmó un petitorio (acción); está en desacuerdo con que los hombres son mejores líderes que las mujeres y tienen prioridad para los empleos (igualdad de género); aprueban la homosexualidad y el divorcio (respeto por los otros); priorizan la libertad de expresión y la participación en las decisiones de gobierno, el trabajo y la comunidad (aspiraciones de libertad) y confían "en la mayoría de la gente" (Jorge, 2015). En el ICPD Refinado sustituyo esta "confianza no especificada" por la "confianza generalizada", que abarca siempre a los extraños y a las personas de otra religión o nacionalidad (Jorge, 2016a, 2017).

Las coincidencias comentadas entre ambas teorías son patentes en nuestra tabla. Como esperaríamos, nuestros índices tienen una fuerte correlación positiva con autonomía y negativa con inmersión. La asociación es menor, pero muy clara, con igualitarismo (positiva) y jerarquía (negativa).

La Figura 5 ilustra gráficamente una de estas relaciones usando el indicador autonomía menos inmersión. Nótese que el puntaje de autonomía es mayor que el de inmersión en todos los países con $36 \%$ o más de la población con valores democráticos arraigados según nuestro índice. 
Las discusiones previas llamaron a prestar atención a las propiedades específicas de cada medida de democracia. Las iniciativas para producir índices y series temporales de datos se han multiplicado junto a la literatura metodológica (Munck, 2009). La democracia puede ser definida operativamente como una variable continua -una cualidad que todos los regímenes poseen en algún grado- o de clasificación, con dos o más categorías (Jorge, 2010:43-4). La evidencia sugiere que la validez y confiabilidad de las escalas de grado es superior en la mayoría de las situaciones (Elkins, 2000).

No hay empero solución obvia al problema de elegir el mejor índice para una aplicación dada. Aunque los existentes tienen altas correlaciones entre sí -pese a su diversidad conceptual y operativa-, "las sutiles diferencias entre ellos pueden y suelen afectar los resultados sustantivos" de investigación (Pemstein et al., 2010).

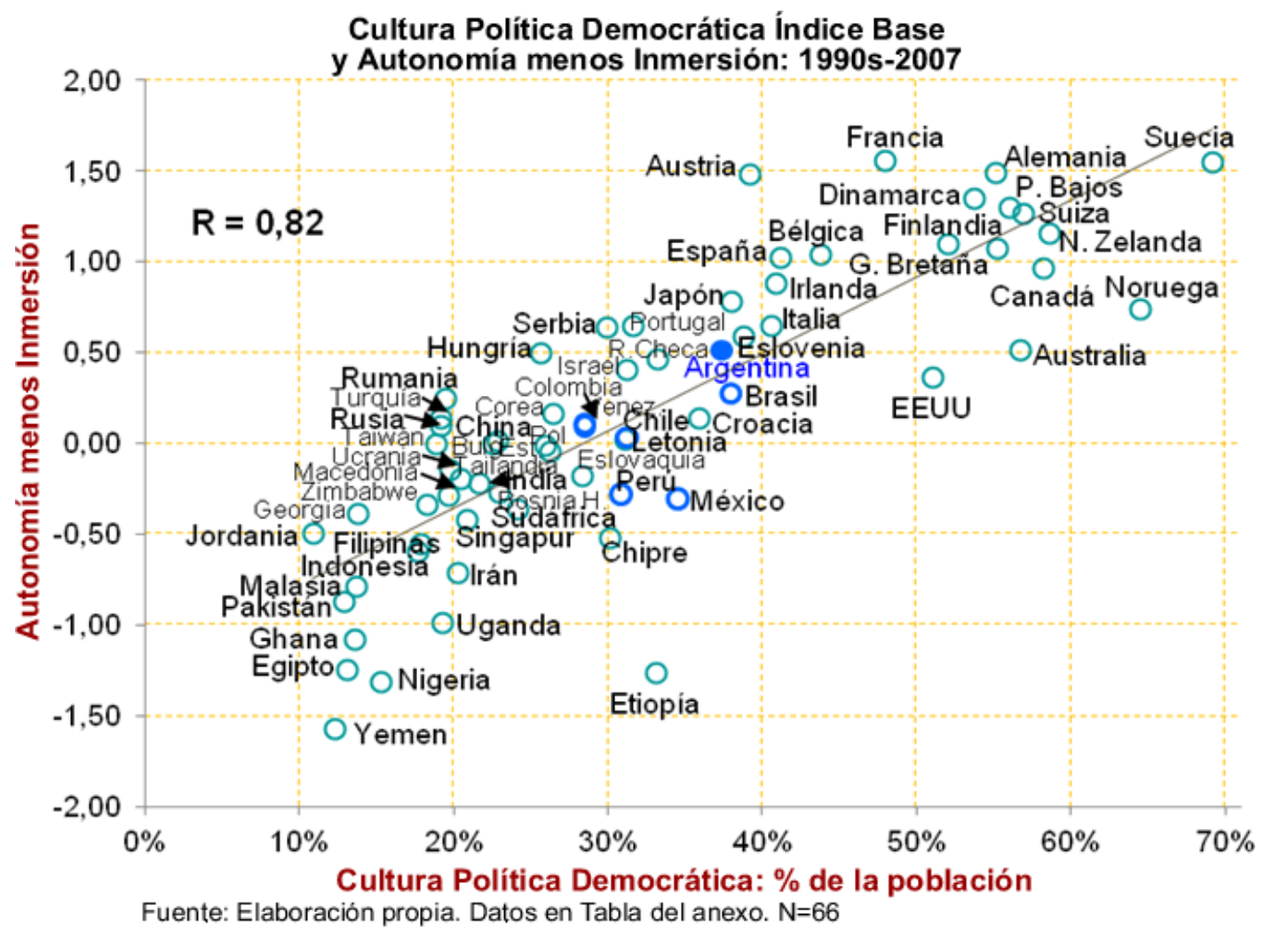

Figura 5

Estas medidas suelen diferir en el tipo y cantidad de sus componentes e indicadores, en el peso que les otorgan y en los criterios de agregación. Las divergencias surgen a menudo de priorizar distintos valores democráticos. Esto es claro en la perspectiva de los "modelos de democracia" (Coppedge y Gerring, 2011; Held, 2006).

Los errores de medición, a veces por sesgos o inconsistencias en el juicio de los expertos, son una fuente seria de perturbación, ya detectada en los dos índices más populares: Polity y 
Freedom House (Armstrong, 2011; Treier y Jackman, 2005). Armstrong vio que la escala 1 a 7 de Freedom House suele dar una nota distinta a países similares, o la misma a países que difieren significativamente. Esto puede ser "sustantiva o estadísticamente relevante" en los estudios comparativos y en los modelos predictivos.

Las medidas que uso en este análisis son las de la Tabla 2. He uniformado todas las escalas tipificándolas en intervalos entre 0 y 1 . Sus valores por país, para los dos periodos estudiados, están en la Tabla del anexo.

El índice de Freedom House (FH) se basa originalmente en dos escalas con sub-puntajes de 040 para derechos políticos y 0-60 para libertades civiles. En el paso final, los dos componentes son recalculados en puntajes 1-7. Notamos ya que este re-escalamiento puede tener un impacto sustantivo (ver también Merkel et al., 2016: 5). Computé un segundo índice FHS, que es la suma de las dos escalas originales. Estos sub-puntajes están disponibles desde 2003.

Índices de Democracia Analizados

\begin{tabular}{|c|c|c|c|c|}
\hline Índice & Descripción & Universo & Periodo & Escala \\
\hline $\begin{array}{l}\text { Democracia Efectiva I (EDI I) } \\
\text { Inglehart y Welzel }\end{array}$ & $\begin{array}{l}\text { FH ó FHS ponderado por Estado de } \\
\text { Derecho y Control de la Corrupción } \\
\text { de los WGI-Banco Mundial }\end{array}$ & $\begin{array}{l}\text { Todos los } \\
\text { paises }\end{array}$ & $\begin{array}{c}\text { Anual:1996 } \\
\text { Presente }\end{array}$ & $\begin{array}{c}\text { Intervalo } \\
0-1\end{array}$ \\
\hline $\begin{array}{l}\text { Democracia Efectiva II (EDI II) } \\
\text { "Logro Democrático": Welzel } \\
\text { (2013) }\end{array}$ & $\begin{array}{l}\text { FH ó FHS ponderado por Derechos } \\
\text { de Integridad Física y Empodera- } \\
\text { miento de CIRI:Cingranelli Richards } \\
\text { Human Rights Data Project }\end{array}$ & $\begin{array}{c}\text { Todos los } \\
\text { paises }\end{array}$ & $\begin{array}{c}\text { Anual:1981 } \\
\text { Presente }\end{array}$ & $\begin{array}{c}\text { Intervalo } \\
0-1\end{array}$ \\
\hline \multirow{2}{*}{$\begin{array}{l}\text { Derechos Politicos y } \\
\text { Libertades Civiles: } \\
\text { Freedom House }\end{array}$} & $\begin{array}{l}\text { Sin sub-puntajes de los com- } \\
\text { ponentes del índice: (FH) }\end{array}$ & $\begin{array}{c}\text { Todos los } \\
\text { paises }\end{array}$ & $\begin{array}{c}\text { Anual: } 1972 \\
\text { Presente }\end{array}$ & $\begin{array}{c}\text { Ordinal } \\
1-7\end{array}$ \\
\hline & $\begin{array}{l}\text { Suma de los sub-puntajes } \\
\text { (FHS) }\end{array}$ & $\begin{array}{c}\text { Todos los } \\
\text { paises }\end{array}$ & $\begin{array}{c}\text { Anual: } 2003 \\
\text { Presente }\end{array}$ & $\begin{array}{c}\text { Intervalo } \\
0-100\end{array}$ \\
\hline $\begin{array}{l}\text { Voz y Rendición de Cuentas } \\
\text { (VAl): Banco Mundial } \\
\text { Kaufmann y Kraay }\end{array}$ & $\begin{array}{l}\text { Participación politica y libertades } \\
\text { civiles. Parte de los Worldwide } \\
\text { Governance Indicators (WGI) }\end{array}$ & $\begin{array}{c}\text { Todos los } \\
\text { paises }\end{array}$ & $\begin{array}{c}\text { Anual:1996 } \\
\text { Presente }\end{array}$ & $\begin{array}{l}\text { Puntajes } \\
\text { Z tipifi- } \\
\text { cados }\end{array}$ \\
\hline $\begin{array}{l}\text { Barómetro de la Democracia. } \\
\text { (DB): Wissenschaftszentrum } \\
\text { Berlin (WZB) y Univ.de Zürich } \\
\text { (UZH) Merkel, Bochsler et al. }\end{array}$ & $\begin{array}{l}\text { Calidad de la Democracia. } 105 \\
\text { indicadores (juicios expertos y } \\
\text { datos fácticos). } 3 \text { dimensiones: } \\
\text { Libertad, Control e lgualdad }\end{array}$ & $\begin{array}{l}\text { Democra- } \\
\text { cias "esta- } \\
\text { blecidas" }\end{array}$ & $\begin{array}{c}\text { Anual:1990 } \\
\text { Presente }\end{array}$ & $\begin{array}{c}\text { Intervalo } \\
0-100\end{array}$ \\
\hline \multirow{2}{*}{ Bertelsmann Stiftung: } & $\begin{array}{l}\text { Índice de Transformación (BTI) } \\
\text { Estado de la Democracia }\end{array}$ & $\begin{array}{l}\text { Paises en } \\
\text { desarrollo }\end{array}$ & $\begin{array}{c}\text { Bianual:2006 } \\
\text { Presente }\end{array}$ & $\begin{array}{c}\text { Intervalo } \\
1-10\end{array}$ \\
\hline & $\begin{array}{l}\text { Gobierno Sustentatble (BSG) } \\
\text { Calidad de la Democracia }\end{array}$ & $\begin{array}{l}\text { Paises de } \\
\text { la OCDE }\end{array}$ & \begin{tabular}{|c|} 
Anual:2014 \\
Presente
\end{tabular} & $\begin{array}{c}\text { Intervalo } \\
1-10\end{array}$ \\
\hline
\end{tabular}

Para el presente trabajo todas las escalas han sido tipificadas en un intervalo 0-1. Valores por pais en la Tabla del anexo. EDI: Effective Democracy Index. VAI: Voice and Accountability Index. DB: Democracy Barometer. BTI: Bertelsmann Transformative Index. BSG: Bertelsmann Sustainable Governance. OCDE: Organización para la Cooperación y el Desarrollo Económico

\section{Tabla 2}

Las dos medidas de Democracia Efectiva son cálculos propios aplicando instrumentos desarrollados por I \& W. Para estos autores, la brecha entre la democracia formal y la real 
tiende a cerrarse a través del estado de derecho, que es el cumplimiento efectivo -no solo el reconocimiento nominal- de las libertades civiles y políticas (Inglehart y Welzel, 2005: 153; Jorge, 2017). La ausencia de corrupción es un aspecto clave, pues la "integridad de la elite" refleja el grado en que ésta se somete a la ley y respeta los derechos de los ciudadanos. La ecuación para el cálculo de EDI I -con todas las escalas entre 0 y 1 - es la siguiente (Alexander et al., 2011):

$E D I I=F H S \times \frac{(R L I+C C I)}{2}$

RLI y CCI son, entre los WGI del Banco Mundial, medidas de estado de derecho (rule of law) y control de la corrupción (Kaufmann et al., 2010).

EDI II se basa en el índice de "logro democrático" de Welzel (2013: 260):

$E D I I I=F H S \times \frac{(P I R+E R)}{2}$

PIR y ER -physical integrity y empowerment rights- son medidas, fundadas en datos fácticos, de los derechos de "integridad física" (tortura, desaparición forzada, ejecuciones extrajudiciales y encarcelamiento político) y de "empoderamiento" (libertad de movimiento, expresión y religión; derechos de los trabajadores y participación política) del proyecto sobre datos de derechos humanos CIRI (Cingranelli y Richards, 2010). Para el periodo de SVS, computé ambos EDIs con el índice FH en lugar de FHS.

Voz y Rendición de Cuentas (VAI) es otro de los índices WGI del Banco Mundial. El Barómetro de la Democracia (DB) mide la "calidad" de las "democracias establecidas" (Merkel et al., 2016). Se distingue de otras escalas por su alto número de indicadores y por incluir datos fácticos, además de evaluaciones de expertos. El índice BTI de la fundación Bertelsmann evalúa, en los países en desarrollo -incluidas las autocracias-, el "estado de la democracia" en su proceso de "transformación" hacia una situación "consolidada". BSG mide la "calidad" democrática de los miembros de la OCDE (1).

Para nuestros datos de la Tabla del anexo, en los dos periodos, las intercorrelaciones entre los EDIs, VAI, BTI y FHS o FH, rondan 0,90 ó más. Las correlaciones de DB con estos índices descienden un poco -pero solo con BTI por debajo de 0,80- y las de BSG promedian 0,77.

La Tabla 3 refleja, para el periodo más reciente 2003-2014, la fuerte asociación de estas medidas de democracia con nuestro ICPD y sus componentes (ver en Jorge, 2015, el análisis de FH). La correlación del índice compuesto ICPD en cada columna -con una pequeña desviación en BTI- es mayor o igual a la de su componente con más poder predictivo. Este último es, en 6 de las 7 columnas, el respeto por los otros. 
Correlaciones entre Cultura Politica Democrática e Índices de Democracia 2003-2014

\begin{tabular}{l|c|c|c|c|c|c|c|c|c} 
& DB & EDI I & \multicolumn{2}{|c|}{ FHS } & EDI II & BSG & VAI & BT & $\mathbf{N}^{*}$ \\
Paises
\end{tabular}

Los datos por pais de los indices ICPD y sus componentes se encuentran en Jorge (2015, 2016a y 2017). Los puntajes de los indices de democracia son promedios trianuales o bianuales centrados en el año en que WVS relevó los datos sobre valores democráticos (puntajes y notas técnicas en Tabla del anexo). (1) Correlación a partir de una aproximación curvilínea cúbica (ver Figura 6) ${ }^{* * *} p<0.001{ }^{* *} p<0.01{ }^{*} p<0.05+p<0.1$ ns: no significativo

Tabla 3

La relación de FHS con los ICPD y los demás valores es no-lineal. Una aproximación cúbica (Figura 6) eleva significativamente la correlación. La curva revela que países con valores democráticos difundidos en menos de $20 \%$ de su población pueden lograr una gama de notas hasta un poco mayores que 6. A partir de este nivel, para obtener notas cada vez más altas, su cultura democrática debe aumentar a tasas crecientes. La relación no-lineal -aquí no mostrada- también se verifica en el caso de FH.

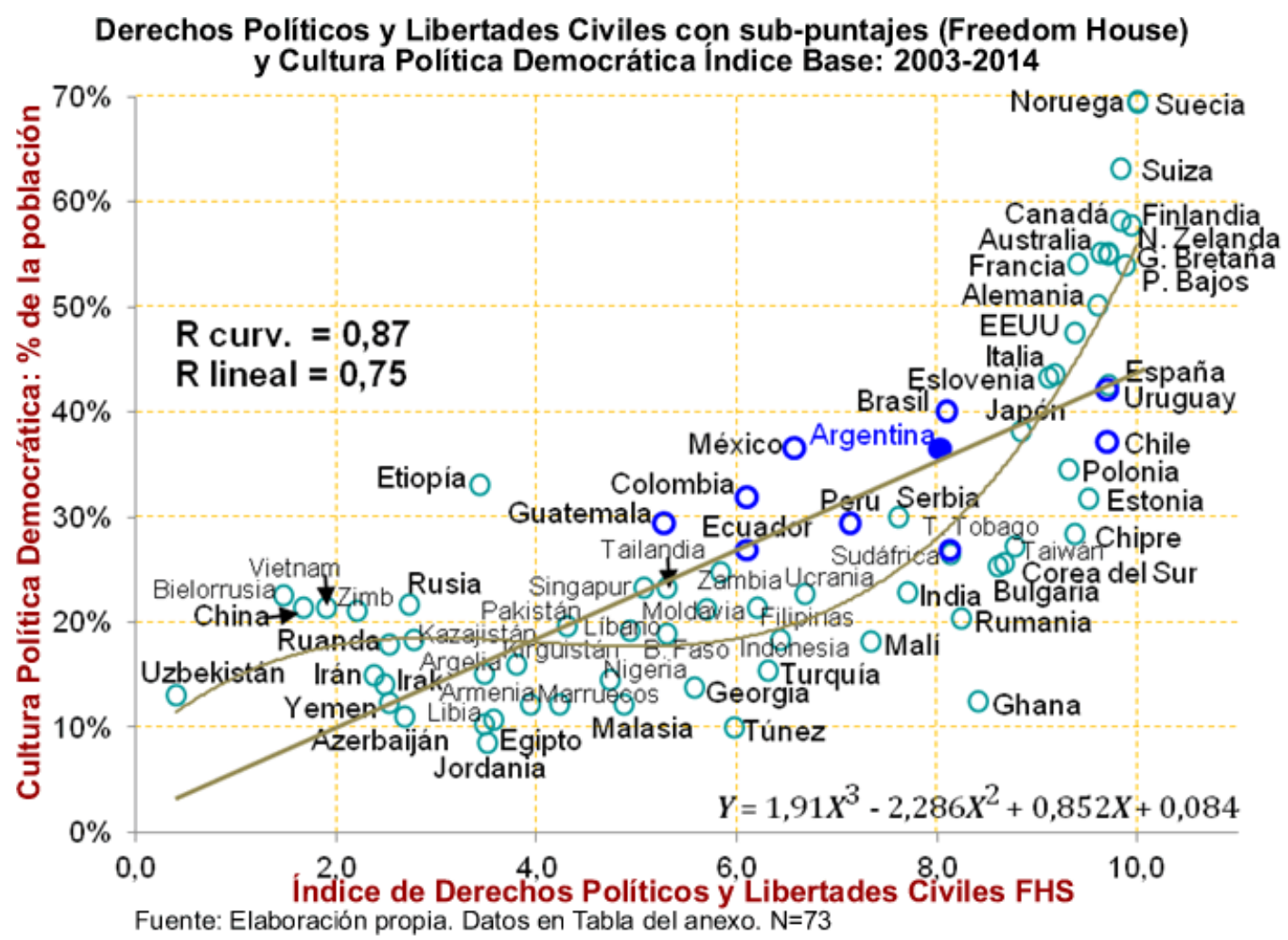

Figura 6 
Al ponderar FHS por el estado de derecho, los índices EDI transforman esa relación curvilínea en lineal (Figuras 7 y 8). Más concretamente, al ser evaluados los países por el criterio más riguroso de la vigencia "efectiva" de los derechos y no solo por el nivel de su democracia formal (el nivel que mide Freedom House según I \& W), es improbable que logren mejorar su calificación en cualquier nivel de la escala sin contar con valores democráticos cada vez más extendidos. La nota promedio de los 73 países cae de 6,4 para FHS a 3,8 (EDI I) y 4,2 (EDI II).

Las medidas de estado de derecho y control de la corrupción del Banco Mundial penalizan con rigor en EDI I a los países latinoamericanos, salvo Uruguay y Chile. Nuestras sociedades se mueven en la Figura 7 a la izquierda de la línea de regresión, reflejando el rezago de su estado de derecho respecto de su cultura democrática e ingreso por habitante (Jorge, 2017; Kaufmann y Kraay, 2002).

Pero si el índice de democracia es ponderado con un énfasis en la vigencia de los derechos humanos, como hacen los indicadores CIRI en EDI II (Figura 8), la nota de la Argentina mejora sustancialmente y se ajusta a lo previsto por su cultura democrática.

Al no incluir a las democracias más consolidadas, las correlaciones de BTI con los valores democráticos son las más bajas de todos los índices (Tabla 3 y Figura 9). Por el contrario, DB evalúa solo a las democracias "establecidas" (Figura 10). El promedio del ICPD Base en estas 36 democracias es $40 \%$, mientras que en los países de BTI es $22 \%$. DB usa criterios rigurosos de evaluación. Su nota promedio en nuestra muestra es 5,3. La de BSG es 6,9.

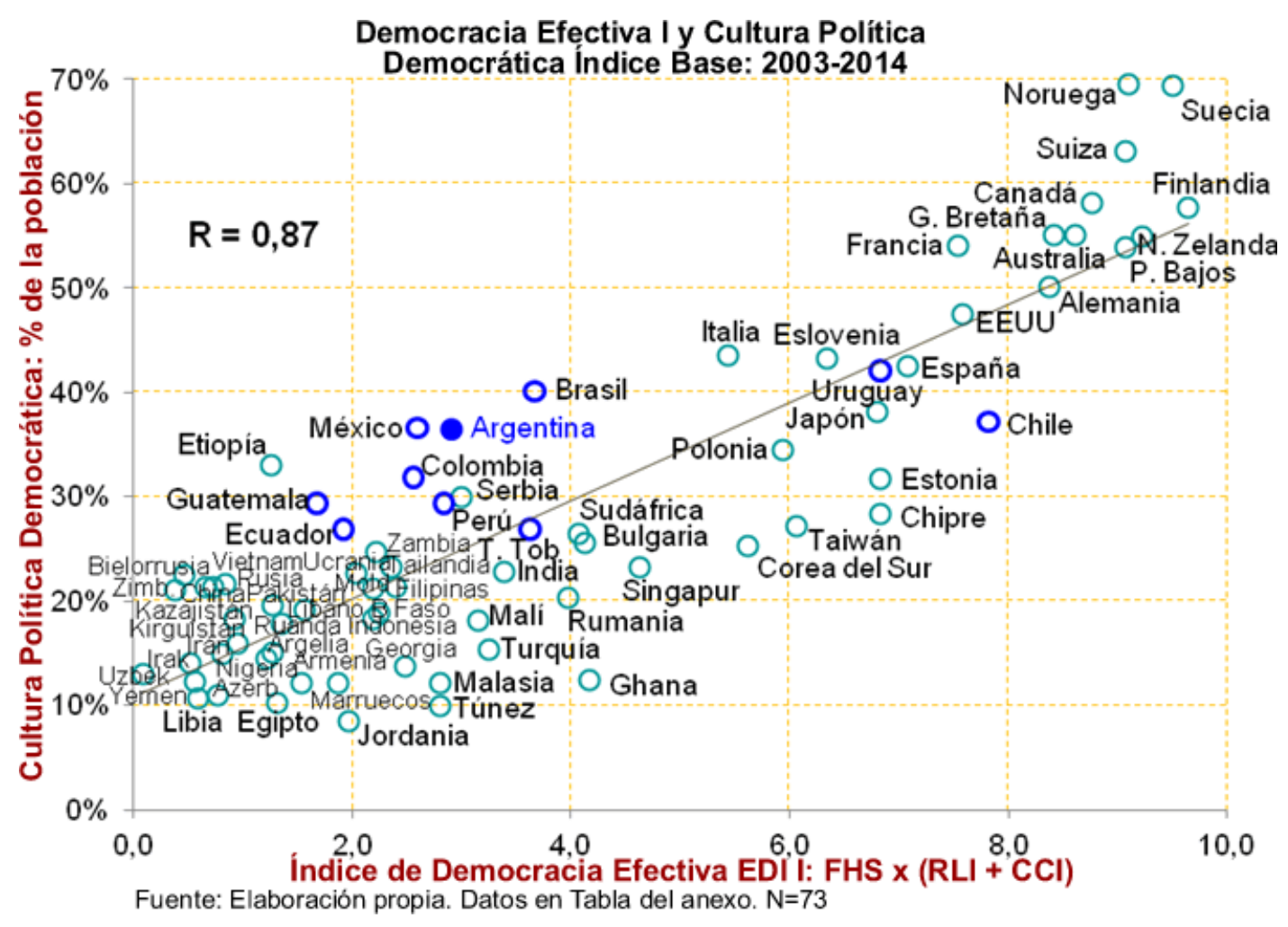

Figura 7 


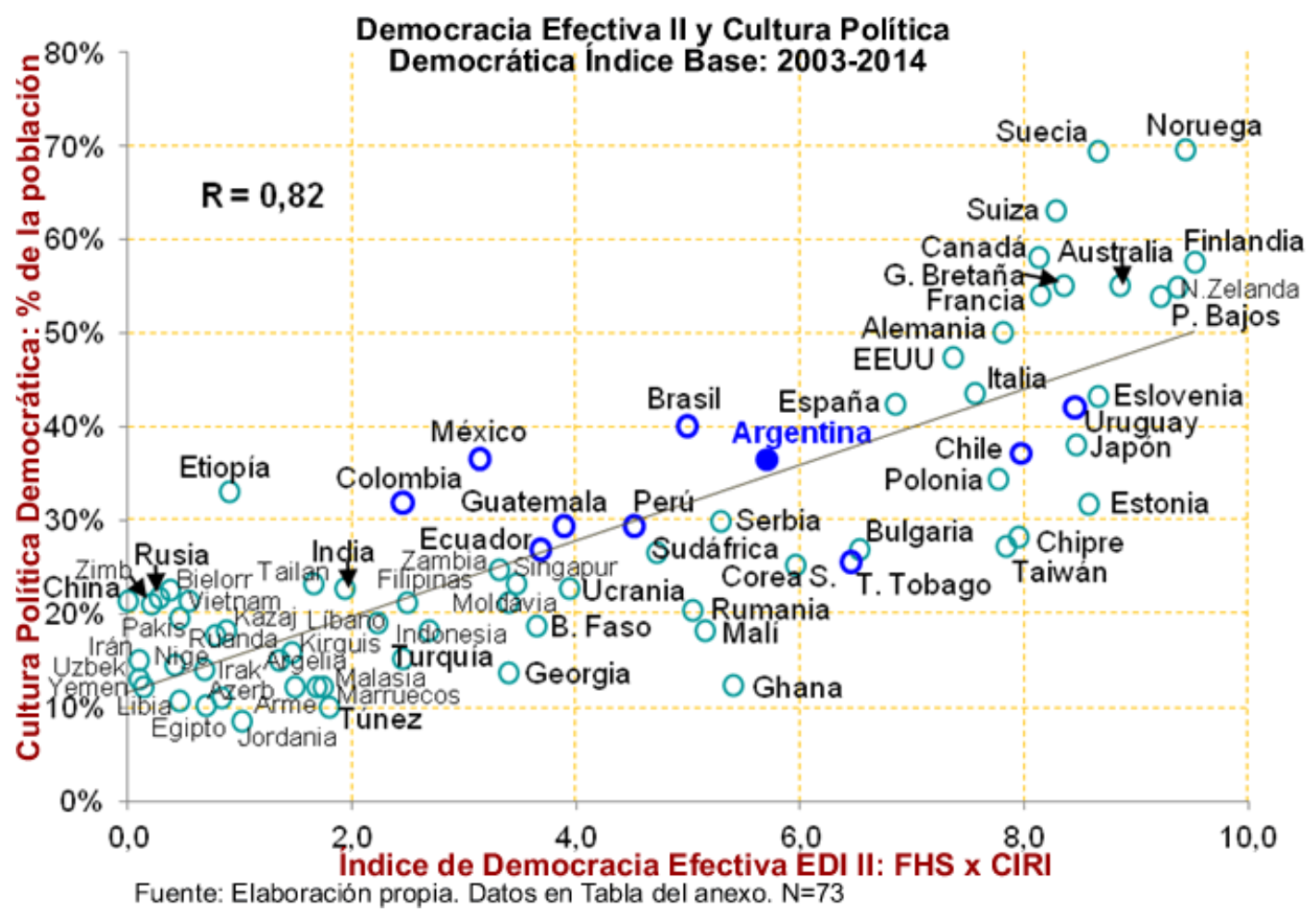

Figura 8

En la teoría de I \& W, el impacto del cambio cultural sobre las instituciones sigue una dinámica "tectónica" (Welzel et al., 2016; Jorge, 2017: 177-8). La difusión creciente de los valores democráticos aumenta gradualmente la "presión" popular por más libertades, hasta alcanzar un umbral o "punto de ruptura" capaz de desencadenar cambios institucionales. Vimos en Jorge (2015) que el $80 \%$ de los países con nota máxima en el índice $\mathrm{FH}$ tenía esos valores difundidos en más del $40 \%$ de la población. Aquí supera este mismo umbral entre el $70 \%$ y el $79 \%$ de los países con nota igual o mayor a 6,5 en EDI I, 7 en EDI II, 8 en VAI, 5 en DB y 6,5 en BSG. 


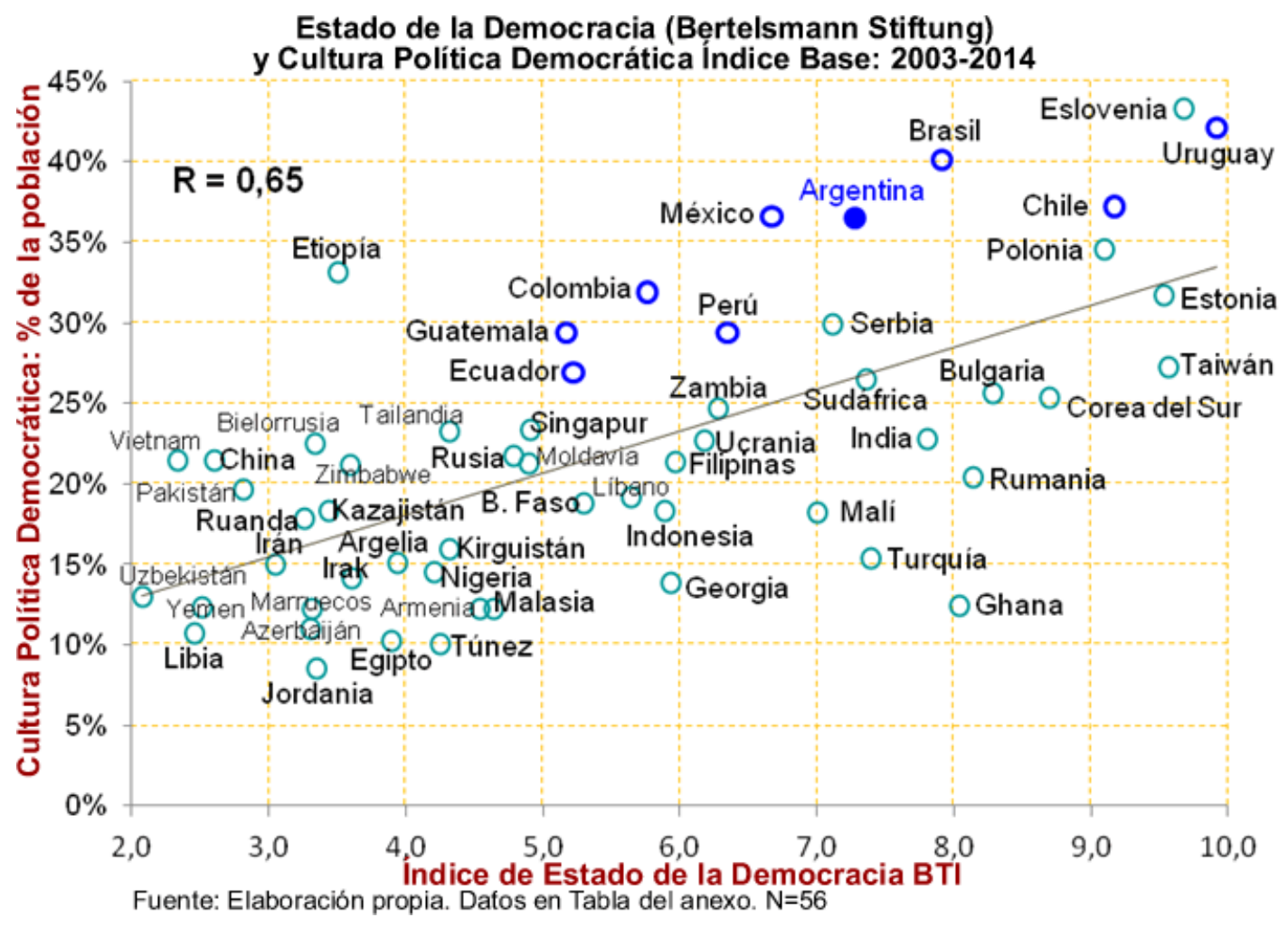

Figura 9

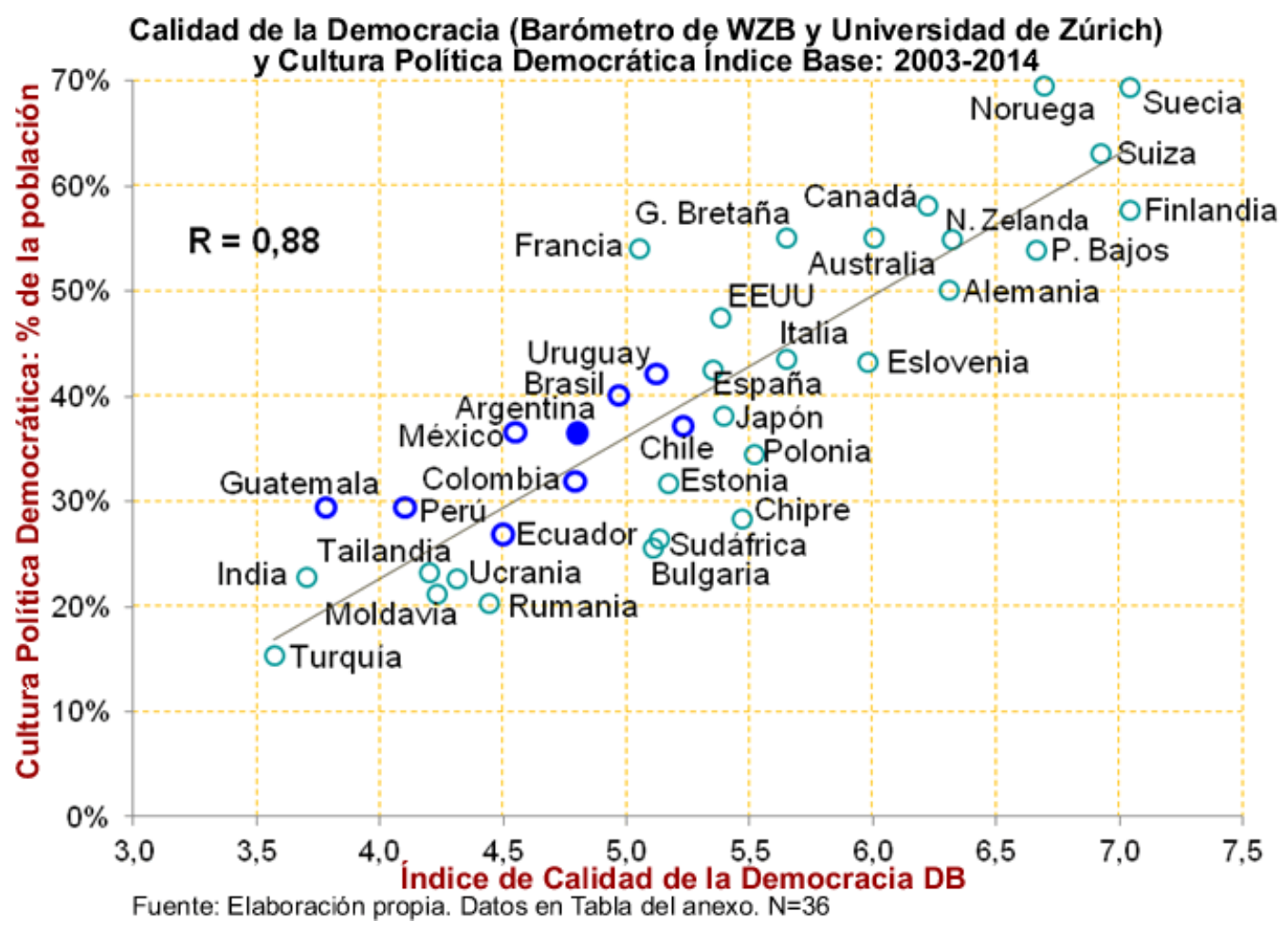

Figura 10 
La relación de los índices de democracia con los valores culturales de Schwartz (Tabla 4) presenta remarcables paralelismos con el examen precedente. Siguiendo el criterio de Licht, Goldschmidt y Schwartz (2007), uso el promedio de cada índice a lo largo de un periodo extenso: desde 1996 -final de la ola más masiva de democratización- y 2007. Sin datos de FHS para todo el periodo, los análisis se basan en $\mathrm{FH}$.

Simplificando, vemos las fuertes correlaciones de estos índices con autonomía menos inmersión, y las menos elevadas, pero claras con la excepción de BTI, con igualdad menos jerarquía.

\begin{tabular}{|c|c|c|c|c|c|c|}
\hline & $\begin{array}{c}\text { EDI } 1 \\
\text { (con FH) }\end{array}$ & VAI & $\begin{array}{c}\text { EDI II } \\
\text { (con FH) }\end{array}$ & FH & DB & BTI \\
\hline Autonomía & $0,79^{\star \star \star \star}$ & $0,77^{\star \star \star}$ & $0,73^{\star \star \star *}$ & $0,72^{\star \star \star}$ & $0,70^{\star * \star *}$ & $0,55^{\star \star * *}$ \\
\hline Autonomía Intelectual & $0,74^{\star \star \star *}$ & $0,74 * * *$ & $0,73^{\star * *}$ & $0,71^{\star \star \star *}$ & $0,58^{\star \star *}$ & $0,61^{\star \star * *}$ \\
\hline Autonomía Afectiva & $0,73^{\star \star *}$ & $0,71^{\star \star *}$ & $0,65^{\star \star \star *}$ & 0,64 *** & $0,65^{* * *}$ & 0,42 ** \\
\hline Igualitarismo & $0,58^{\star * *}$ & 0,56 *** & $0,52^{* * *}$ & 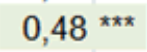 & $0,48 * * \star$ & $0,12 \mathrm{~ns}$ \\
\hline Armonía & $0,39^{\star \star \star}$ & $0,44^{\star \star \star *}$ & $0,47^{* * *}$ & $0,43^{\star \star *}$ & $0,25+$ & 0,41 ** \\
\hline Inmersión & $-0,82^{\star * \star}$ & $-0,81^{\star * *}$ & $-0,78^{\star * *}$ & $-0,766^{\star \star \star}$ & $-0,72^{\star * *}$ & $-0,64^{\star * *}$ \\
\hline Jerarquía & $-0,55^{\star \star *}$ & $-0,58^{\star * \star}$ & $-0,59 * \star \star x$ & $-0,54 * * *$ & $-0,61 \approx * *$ & $-0,35^{\star \star}$ \\
\hline Dominio & $-0,06$ & $-0,09 * \star *$ & $-0,12$ & $-0,05$ & $-0,27+$ & $-0,13 \mathrm{~ns}$ \\
\hline Autonomía menos Inmersión & $0,82^{\star \star \star *}$ & $0,81^{\star \star \star}$ & $0,77^{\star \star \star}$ & $0,75^{\star \star \star}$ & $0,73^{* * \star}$ & $0,62^{\star \star *}$ \\
\hline Igualitarismo menos Jerarquía & 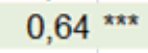 & $0,65^{\star \star * *}$ & $0,64 * \star * \star$ & 0,59 *** & $0,63^{* * *}$ & $0,34^{\star *}$ \\
\hline Armonía menos Dominio & $0,32^{\star * *}$ & $0,38^{\star \star *}$ & $0,41^{\star \star *}$ & $0,35^{\star \star \star}$ & 0,29 * & 0,35 ** \\
\hline$N^{\circ}$ de Paises & 75 & 75 & 75 & 75 & 47 & 51 \\
\hline
\end{tabular}

Los puntajes de los índices de democracia son un promedio de todo el periodo 1996-2007 (criterio empleado por Licht, Goldschmidt y Scwartz, 2007). Datos en Tabla del anexo. ${ }^{\star \star \star} p<0.001{ }^{\star \star} p<0.01{ }^{\star} p<0.05+p<0.1 \mathrm{~ns}$ : no significativo

Tabla 4

EDI I refleja otra vez el retraso del estado de derecho en América Latina, aquí respecto de los valores de autonomía prevalecientes (Figura 11). También surgen umbrales. Por ejemplo, el 86\% de los países con nota 7 o más en EDI I tienen puntajes de autonomía superiores en 0,50 a los de inmersión. Aunque menos acentuada que en el caso anterior, la relación de autonomía menos inmersión con $\mathrm{FH}$ es asimismo no-lineal: la correlación cuadrática es 0,79 y la lineal 0,75 .

\section{Modelos predictivos}

A continuación pongo a prueba la relación entre los valores de las dos teorías y el gobierno democrático mediante modelos de regresión lineal multivariados que predicen todos los índices examinados de democracia. 
EI ICPD Base -disponible para más países que el refinado- es usado como predictor en un conjunto de modelos del periodo 2003-2014; los valores de Schwartz, en otro conjunto del periodo 1996-2007. En ambos casos se incluyen entre los predictores el ingreso por habitante; el nivel de desarrollo humano; la distribución del ingreso; los años de democracia sin interrupciones; la religión predominante; la diversidad étnica, lingüística y religiosa; y el origen colonial (Jorge, 2016b, 2017).

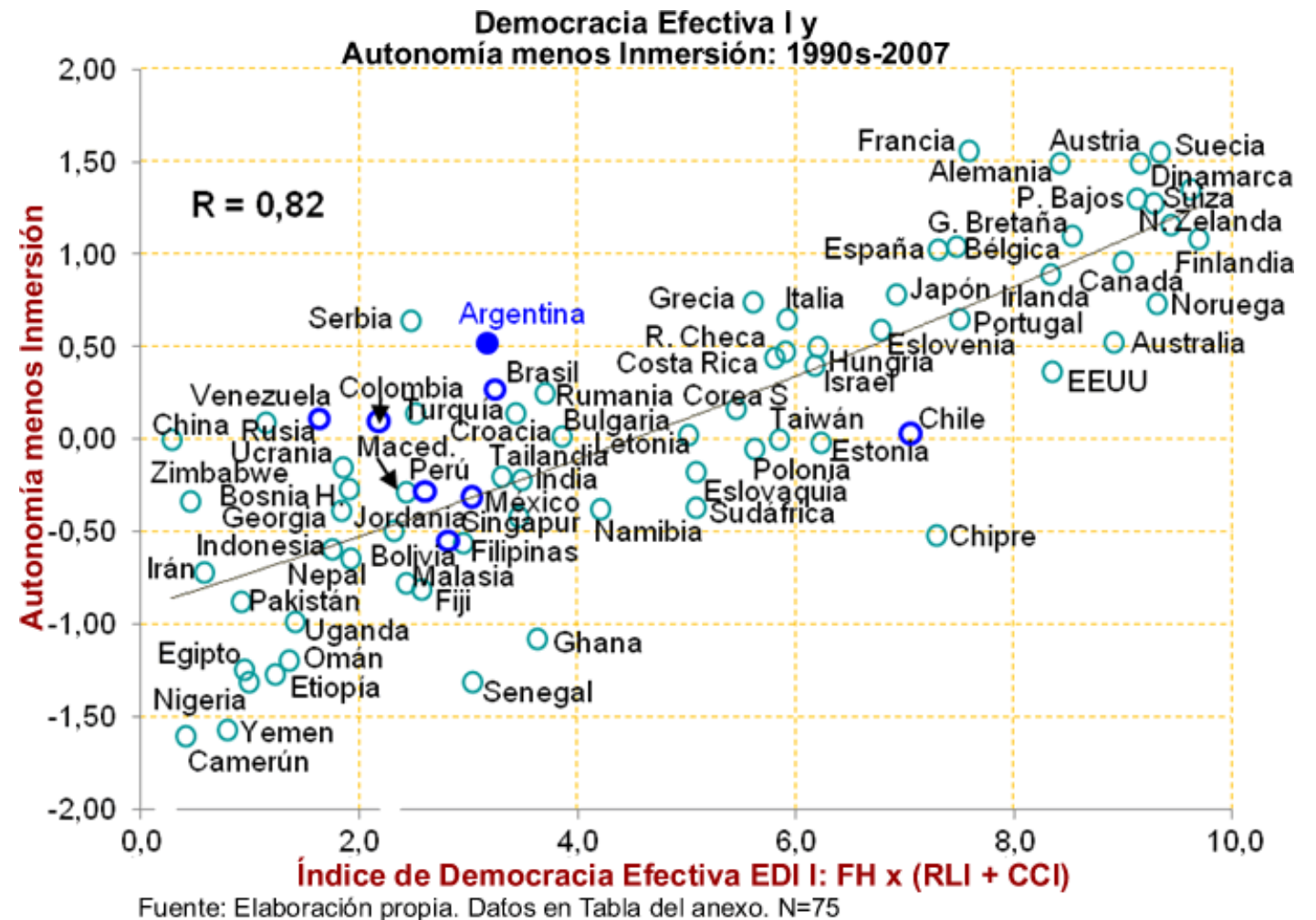

Figura 11

Las tablas siguientes presentan los resultados más importantes. Conforme con la teoría, en los 10 modelos de las Tablas 5 y 6 el ICPD es un predictor altamente significativo de los diferentes índices de democracia, junto con el desarrollo humano medido por el PNUD -que engloba ingreso, salud y educación- o el ingreso per cápita.

Una vez incorporadas estas potentes variables, la significación de la religión, la diversidad y el origen colonial desaparece o es esporádica y débil. La influencia de la experiencia democrática (medida en años) emerge solo en 3 de los modelos. En otro -el de BSG, con países de la OCDE- lo hace con un paradójico signo negativo. Esto solo refleja el hecho de que en esta muestra, manteniendo constantes la cultura democrática y el desarrollo humano, países como Finlandia, Estonia, Chile o Eslovenia tienen igual o mejor desempeño que otros con más años 
de democracia. La inserción activa en organizaciones voluntarias es marginalmente significativa en los modelos de FHS y VAI.

En los análisis de las Tablas 7 y 8, siguiendo la estrategia de Licht et al. (2007), introduje en cada regresión el perfil cultural completo de los países en las tres dimensiones de Schwartz, usando una sola orientación polar por vez para evitar problemas de colinealidad.

La autonomía (inmersión con coeficiente negativo) y el igualitarismo (o jerarquía con signo negativo) son claros predictores de todos los índices de democracia. En algunos modelos alternativos es significativa la armonía (o el dominio negativamente).

\section{Análisis de Regresión: Cultura Politica y Democracia 2003-2014}

Variables
Independientes
ICPD Base: Cultura
Politica Democrática
Años de Democracia
sin Interrupciones
İndice de Desarrollo
Humano IDH-PNUD
Ingreso p/hab PPA
Logaritmo natural
Diversidad
Étnica
Diversidad
Lingüistica
Miembros Activos
de Org. Voluntarias
Constante
$\mathrm{R}^{2}$ Ajustada
$\mathrm{N}^{0}$ de Casos

Democracia Efectiva EDI I: FHS x (RLI+CCl)

\begin{tabular}{c|c} 
Modelo I & Modelo II \\
$0,895^{\star * \star}$ & $0,895^{\star \star \star}$
\end{tabular}

$(4,567)$

0,002 **

$(4,437)$

$(2,581)$

0,484 *ᄎ*

$(3,455)$

\begin{tabular}{|c|c|}
$(3,455)$ & $0,070 *$ \\
& $(3,186)$ \\
& $-0,167+$ \\
& $(-1,945)$ \\
& 0,151 * \\
& $(1,970)$ \\
& \\
& \\
$-0,294$ ** & $-0,588$ ** \\
$(-3,152)$ & $(-2,695)$ \\
$\mathbf{0 , 8 1}$ & $\mathbf{0 , 8 1}$ \\
72 países & 70 países
\end{tabular}

Dem. Efec- F tiva EDI II: FHS $x$ CIRI

$1,395 * * \star$

$(8,444)$ puntajes FHS

\section{Modelo I Modelo II}

0,856 ***

0,907
$(4,375)$

0,492 **

$(2,644)$

0,589 **
$(2,572)$

Fuente: Cálculos propios. Las entradas son coeficientes de regresión no estandarizados (valores t entre paréntesis). Casos: Paises de la Tabla del anexo, periodo WVS (2003-2014). Variables dependientes: Escala 0-1. Ver notas técnicas del anexo. Variables Independientes: Datos centrados en el año WVS en que se relevaron los valores de ICPD (promedio trianual) o más próximos a esa fecha. IDH-PNUD: Escala 0-1. Ingreso por Habitante (logarit-mo na-tural). Banco Mundial, en dólares interna-cionales constantes de 2011, paridad de poder ad-quisitivo. Gini: Escala 0-1. Datos SWIID (F. Solt), excepto en el modelo de BSG (datos OCDE). Diversidad Étnica, Linguística y Religiosa: Escalas 0-1. Índices de Fraccionalización de Alesina et al. (2003). Religión predominante: Variable dummy con datos de Alesina et al. (2003). Años de Democracia sin Interrupciones: Contados desde 1900 hasta el año WVS (excepto modelo de BSG, hasta 2016) según la base del Proyecto Polity. Miembros Activos de Organizaciones Vo-luntarias Proporción de la población. Escala 0 -1. Datos de WVS.. ${ }^{* \star *} p<0.001{ }^{* *} p<0.01{ }^{*} p<0.05 t p<0.1$ ns: no significativo

Tabla 5 
El desarrollo humano pierde significación en los modelos de DB y BTI. Comparando con el análisis del ICPD, en estas regresiones crece en importancia la diversidad lingüística o religiosa -positivamente asociada con la democracia cuando se mantienen constantes los valores de autonomía, igualitarismo y armonía-, así como la experiencia democrática. Solo esporádicamente influyen la distribución del ingreso, el protestantismo y el legado colonial británico.

Ante los complejos desafíos que enfrenta la democracia en todo el mundo, en especial en América Latina, surge un mensaje teórica y empíricamente fundado. La libertad individual con un sentido altruista, el igualitarismo, la preocupación y el respeto por los demás, la confianza generalizada y la participación, son los valores a promover para defender y profundizar la vida democrática y el estado de derecho.

\begin{tabular}{|c|c|c|c|c|c|}
\hline \multirow{3}{*}{$\begin{array}{l}\text { Variables } \\
\text { Independientes }\end{array}$} & \multirow{2}{*}{\multicolumn{2}{|c|}{$\begin{array}{l}\text { Voz y Rendición de } \\
\text { Cuentas VAl }\end{array}$}} & \multirow{3}{*}{$\begin{array}{l}\text { Calidad de la } \\
\text { Dem. DB } \\
\text { WZB-UZH }\end{array}$} & \multicolumn{2}{|c|}{ Bertelsmann Stiftung } \\
\hline & & & & \multirow{2}{*}{$\begin{array}{l}\text { BTl: Estado } \\
\text { de la Dem. }\end{array}$} & \multirow{2}{*}{$\begin{array}{c}\text { BSG: } \\
\text { Calidad de } \\
\text { la Dem. }\end{array}$} \\
\hline & Modelo I & Modelo II & & & \\
\hline $\begin{array}{l}\text { ICPD Base: Cultura } \\
\text { Politica Democrática }\end{array}$ & $\begin{array}{c}0,8122^{\star \star \star} \\
(4,829)\end{array}$ & $\begin{array}{l}0,877^{\star \star \star *} \\
(5,627)\end{array}$ & $\begin{array}{l}0,300 \text { ** } \\
(3,446)\end{array}$ & $\begin{array}{l}0,816^{* *} \\
(2,975)\end{array}$ & $\begin{array}{l}0,969 \text { *** } \\
(4,352)\end{array}$ \\
\hline $\begin{array}{l}\text { Años de Democracia } \\
\text { sin Interrupciones }\end{array}$ & & & & $\begin{array}{l}0,007^{\star \star \star} \\
(4,346)\end{array}$ & $\begin{array}{l}-0,002 \text { * } \\
(-2,294)\end{array}$ \\
\hline $\begin{array}{l}\text { Índice de Desarrollo } \\
\text { Humano IDH-PNUD }\end{array}$ & $\begin{array}{l}0,594^{\star \star \star} \\
(3,513)\end{array}$ & & $\begin{array}{l}0,354 \text { ** } \\
(3,104)\end{array}$ & & $\begin{array}{c}0,961+ \\
(1,778)\end{array}$ \\
\hline $\begin{array}{l}\text { Ingreso p/hab PPA } \\
\text { Logaritmo natural }\end{array}$ & & $\begin{array}{l}0,072^{\star \star \star *} \\
(3,421)\end{array}$ & & $\begin{array}{l}0,047 * \\
(2,132)\end{array}$ & \\
\hline $\begin{array}{l}\text { Diversidad } \\
\text { Religiosa }\end{array}$ & & & & $\begin{array}{l}0,154+ \\
(1,898)\end{array}$ & \\
\hline $\begin{array}{l}\text { Religión Predomi- } \\
\text { nante Protestante }\end{array}$ & & & $\begin{array}{l}0,041 \text { * } \\
(2,065)\end{array}$ & & \\
\hline $\begin{array}{l}\text { Miembros Activos } \\
\text { de Org. Voluntarias }\end{array}$ & $\begin{array}{c}0,177 * \\
(1,981)\end{array}$ & $\begin{array}{l}0,148+ \\
(1,737)\end{array}$ & & & \\
\hline Constante & $\begin{array}{c}-0,162 \text { ns } \\
(-1,459)\end{array}$ & $\begin{array}{l}-0,411 \text { * } \\
(-2,228)\end{array}$ & $\begin{array}{l}0,110 \mathrm{~ns} \\
(1,524)\end{array}$ & $\begin{array}{l}-0,198 \mathrm{~ns} \\
(-0,992)\end{array}$ & $\begin{array}{l}-0,447 \text { ns } \\
(-1,067)\end{array}$ \\
\hline $\mathbf{R}^{2}$ Ajustada & 0,69 & 0,68 & 0,82 & 0,59 & 0,69 \\
\hline $\mathrm{N}^{0}$ de Casos & 79 países & 80 países & 36 paises & 55 países & 28 países \\
\hline
\end{tabular}


Análisis de Regresión: Valores Culturales y Democracia 1990s-2007

\begin{tabular}{|c|c|c|c|c|}
\hline \multirow{2}{*}{$\begin{array}{l}\text { Variables } \\
\text { Independientes }\end{array}$} & \multirow{2}{*}{$\begin{array}{c}\text { Democracia } \\
\text { Efectiva I } \\
\mathrm{FH} \times(\mathrm{RLI}+\mathrm{CCl})\end{array}$} & \multicolumn{2}{|c|}{$\begin{array}{c}\text { Democracia Efectiva II } \\
\text { FH } \times \text { CIRI }\end{array}$} & \multirow{2}{*}{$\begin{array}{l}\text { Freedom } \\
\text { House FH }\end{array}$} \\
\hline & & Modelo I & Modelo II & \\
\hline Inmersión & $\begin{array}{l}-0,219 \text { ** } \\
(-3,024)\end{array}$ & $\begin{array}{l}-0,207^{\star} \\
(-2,161)\end{array}$ & & $\begin{array}{l}-0,230 \text { * } \\
(-2,466)\end{array}$ \\
\hline Igualitarismo & $\begin{array}{l}0,175^{\text {** }} \\
(2,731)\end{array}$ & & & \\
\hline Jerarquía & & $\begin{array}{l}-0,176 \text { *** } \\
(-3,338)\end{array}$ & $\begin{array}{l}-0,139 * \\
(-2,290)\end{array}$ & $\begin{array}{l}-0,1199^{*} \\
(-2,328)\end{array}$ \\
\hline \multicolumn{5}{|l|}{ Dominio } \\
\hline Armonia & & & $\begin{array}{l}0,205 \text { ** } \\
(2,553)\end{array}$ & \\
\hline $\begin{array}{l}\text { Años de Democracia } \\
\text { sin Interrupciones }\end{array}$ & $\begin{array}{l}0,001+ \\
(1,832)\end{array}$ & $\begin{array}{l}0,0022^{\star \star} \\
(2,770)\end{array}$ & $\begin{array}{l}0,003^{* * *} \\
(4,576)\end{array}$ & $\begin{array}{l}0,002 \text { * } \\
(2,071)\end{array}$ \\
\hline $\begin{array}{l}\text { Índice de Desarrollo } \\
\text { Humano IDH-PNUD }\end{array}$ & $\begin{array}{l}0,532 * * \\
(3271)\end{array}$ & $\begin{array}{r}0,562 \\
(2430)\end{array}$ & $\begin{array}{l}0,805^{* * *} \\
(4,332)\end{array}$ & $0,392+$ \\
\hline $\begin{array}{l}\text { Distribución del } \\
\text { Ingreso: Gini SWIID }\end{array}$ & $\begin{array}{l}-0,473 \text { ** } \\
(-3,027)\end{array}$ & & & \\
\hline $\begin{array}{l}\text { Diversidad } \\
\text { Religiosa }\end{array}$ & & & $\begin{array}{l}0,145+ \\
(1,783)\end{array}$ & \\
\hline $\begin{array}{l}\text { Origen Colonial } \\
\text { Británico }\end{array}$ & $\begin{array}{l}0,079 \text { * } \\
(2,316)\end{array}$ & & & \\
\hline $\begin{array}{l}\text { Religión Predomi- } \\
\text { nante: Protestante }\end{array}$ & $\begin{array}{l}0,139 \text { *ᄎ* } \\
(3,374)\end{array}$ & & & \\
\hline Constante & $\begin{array}{l}0,164 \mathrm{~ns} \\
(0,320)\end{array}$ & $\begin{array}{l}1,243 \text { * } \\
(2,459)\end{array}$ & $\begin{array}{c}-0,724 \text { ns } \\
(-1,609)\end{array}$ & $\begin{array}{l}1,532 \text { ** } \\
(3,118)\end{array}$ \\
\hline $\mathbf{R}^{2}$ Ajustada & 0,85 & 0,72 & 0,74 & 0,64 \\
\hline $\mathrm{N}^{0}$ de Casos & 74 países & 74 países & 73 países & 74 paises \\
\hline
\end{tabular}

Fuente: Cálculos propios. Las entradas son coeficientes de regresión no tipificados (valo. res $\mathbf{t}$ entre paréntesis). Casos: Paises de la Tabla del anexo. Variables dependientes: Escala 0-1.Ver notas técnicas del anexo.. Variables independientes. Promedio mediados 1990s-2007. Origen Colonial: Variables dummy con datos de Hoover \& Pérez, (2004) y Price (2003). Años de Democracia sin Interrupciones: contados desde 1900 hasta 2007 según la base del Proyecto Polity. En caso de una o más interrupciones dentro del periodo 1996-2007, se registra el número mayor ${ }^{* * *} p<0.001{ }^{* *} p<0.01{ }^{*} p<0.05 \dagger$ $\mathrm{p}<0.1 \mathrm{~ns}$ : no significativo 
Análisis de Regresión: Valores Culturales y Democracia 1990s-2007 (continuación)

\begin{tabular}{|c|c|c|c|c|c|}
\hline \multirow{2}{*}{$\begin{array}{l}\text { Variables } \\
\text { Independientes }\end{array}$} & \multicolumn{2}{|c|}{$\begin{array}{l}\text { Voz y Rendición de } \\
\text { Cuentas VAI }\end{array}$} & \multirow{2}{*}{$\begin{array}{c}\text { Calidad de la } \\
\text { Dem. DB } \\
\text { WZB-UZH }\end{array}$} & \multicolumn{2}{|c|}{$\begin{array}{l}\text { Bertelsmann Stiftung } \\
\text { Estado de la Dem. BTI }\end{array}$} \\
\hline & Modelo I & Modelo II & & Modelo I & Modelo II \\
\hline Inmersión & $\begin{array}{l}-0,179^{\star \star} \\
(-3,132)\end{array}$ & & $\begin{array}{l}-0,078^{*} \\
(-2,120)\end{array}$ & $\begin{array}{l}-0,548^{\star \star \star} \\
(-6,672)\end{array}$ & $\begin{array}{l}-0,569 \text { *ᄎ夫 } \\
(-6,229)\end{array}$ \\
\hline Igualitarismo & & & $\begin{array}{l}0,060+ \\
(1,926)\end{array}$ & & \\
\hline Jerarquía & $\begin{array}{l}-0,1144^{\star \star \star} \\
(-3,676)\end{array}$ & $\begin{array}{l}-0,082 \text { * } \\
(-2,182)\end{array}$ & & & $\begin{array}{l}-0,1744^{\star \star} \\
(-2,985)\end{array}$ \\
\hline Dominio & & & $\begin{array}{l}-0,110 \text { * } \\
(-2,270)\end{array}$ & $\begin{array}{l}-0,381 \text { ** } \\
(-2,736)\end{array}$ & \\
\hline Armonía & & $\begin{array}{l}0,103 \text { * } \\
(2,189)\end{array}$ & & & \\
\hline $\begin{array}{l}\text { Años de Democracia } \\
\text { sin Interrupciones }\end{array}$ & $\begin{array}{l}0,001 * \star \star * \\
(2,773)\end{array}$ & 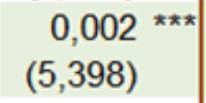 & & & \\
\hline $\begin{array}{l}\text { Índice de Desarrollo } \\
\text { Humano PNUD }\end{array}$ & $\begin{array}{l}0,537^{\star \star *} \\
(3,825)\end{array}$ & $\begin{array}{c}0,744 \text { *** } \\
(5,836)\end{array}$ & & & \\
\hline $\begin{array}{l}\text { Distribución del } \\
\text { Ingreso: Gini SWIID }\end{array}$ & & & $\begin{array}{l}-0,358^{\star \star \star *} \\
(-4,404)\end{array}$ & & \\
\hline $\begin{array}{l}\text { Diversidad } \\
\text { Linguïstica }\end{array}$ & $\begin{array}{l}0,108 \text { * } \\
(2,203)\end{array}$ & $\begin{array}{c}0,084+ \\
(1,681)\end{array}$ & & & $\begin{array}{l}0,191 * \\
(2,119)\end{array}$ \\
\hline $\begin{array}{l}\text { Diversidad } \\
\text { Religiosa }\end{array}$ & & & & $\begin{array}{l}0,164+ \\
(1,747)\end{array}$ & \\
\hline $\begin{array}{l}\text { Origen Colonial } \\
\text { Británico }\end{array}$ & & & $\begin{array}{l}0,048^{\star \star} \\
(2,881)\end{array}$ & & \\
\hline $\begin{array}{l}\text { Religión Predomi- } \\
\text { nante: Protestante }\end{array}$ & & & $\begin{array}{c}0,066^{\star * \star} \\
(4,464)\end{array}$ & & \\
\hline Constante & $\begin{array}{l}1,120 \\
(3,739)\end{array}$ & $\begin{array}{l}-0,224 \mathrm{~ns} \\
(-0,801)\end{array}$ & $\begin{array}{l}1,0666^{\star \star} \\
(3,180)\end{array}$ & $\begin{array}{l}4,259^{\star \star \star} \\
(6,164)\end{array}$ & $\begin{array}{l}3,278^{\star \star \star} \\
(8,771)\end{array}$ \\
\hline $\begin{array}{l}R^{2} \text { Ajustada } \\
N^{0} \text { de Casos }\end{array}$ & $\begin{array}{c}\mathbf{0 , 8 1} \\
73 \text { países }\end{array}$ & $\begin{array}{c}\mathbf{0 , 7 9} \\
73 \text { países }\end{array}$ & $\begin{array}{c}\mathbf{0 , 8 0} \\
47 \text { países }\end{array}$ & $\begin{array}{c}\mathbf{0 , 4 9} \\
50 \text { países }\end{array}$ & $\begin{array}{c}\mathbf{0 , 5 0} \\
50 \text { países }\end{array}$ \\
\hline
\end{tabular}

Tabla 8

\section{Notas}

(1) La metodología, datos y demás documentación de los índices BTI y BSG son accesibles en http://bertelsmannstiftung.de

\section{Bibliografía}


Alesina, A.; Devleeschawer, A.; Easterly, W.; Kurlat, S. y Wacziarg, R. (2003). Fractionalization. Journal of Economic Growth, 8, pp. 155-194.

Alexander, A.; Inglehart, R. y Welzel, C. (2011). Measuring effective democracy: A defense. International Political Science Review, 33(1), pp. 41-62.

Almond, G. y Verba, S. (eds.). (1989). The Civic Culture Revisited. Newbury Park: Sage.

Almond, G. y Verba, S. (1963). The Civic Culture. Newbury Park: Sage.

Armstrong, D. A. (2011). Stability and change in the Freedom House political rights and civil liberties measures. Journal of Peace Research, 48(5), pp. 653-662.

Art, D. (2012). What Do We Know About Authoritarianism After Ten Years? Comparative Politics, 44(3), pp. 351-73.

Baldwin, R. (2016). The Great Convergence. Cambridge: The Belknap Press of Harvard University Press.

Bertelsmann Stiftung (2016). BTI 2016. Global Results. Recuperado de http://bertelsmannstiftung.de

Bollen, K. A. y Paxton, P. (2000). Subjective Measures of Liberal Democracy. Comparative Political Studies, 33(1), pp. 58-86.

Cingranelli, D. L. y Richards, D. L. (2010). The Cingranelli and Richards (CIRI) Human Rights Data Project. Human Rights Quarterly, 32(2), pp. 401-24.

Clark, W.; Golder, M. y Golder, S. N. (2013). Principles of Comparative Politics. Thousand Oaks: SAGE.

Coppedge, M. (2012). Democratization and Research Methods. Cambridge: Cambridge University Press.

Coppedge, M. y Gerring, J. (2011). Conceptualizing and Measuring Democracy: A New Approach. Perspective on Politics, 9(2), pp. 247-67.

Dahlum, S. y Knutsen, C. H. (2016). Democracy by Demand? British Journal of Political Science, 47(2), pp. 437-61.

Dalton, R. y Welzel, C. (eds.). (2014). The Civic Culture Transformed. New York: Cambridge University Press.

Delfino, G. y Beramendi, M. (2015). Un análisis sobre la confianza y el interés cívico en Argentina: estudio de los datos de la World Values Survey 1984-2013. Anuario de Investigaciones/Facultad de Psicología UBA, XXI, pp. 189-96.

Diamond, L. J. y Plattner, M. F. (eds.) (2015). Democracy in Decline? Baltimore: John Hopkins University Press.

Diamond, L. J. y Morlino, L. (2004). The Quality of Democracy: An Overview. Journal of Democracy, 15(4), pp. 20-31. 
Dobewall, H. y Rudnev, M. (2014). Common and Unique Features of Schwartz's and Inglehart's Value Theories at the Country and Individual Levels. Cross-Cultural Research, 48(1), pp. 45-77.

Easton, D. (1965). A Systems Analysis of Political Life. New York: John Wiley and Sons.

Eckstein, H. (1966). Division and Cohesion in Democracy. Princeton: Princeton University Press.

Elkins, Z. (2000). Gradations of democracy? Empirical tests of alternative conceptualizations. American Journal of Political Science, 44(2), pp. 287-94.

Ercan, S. A. y Gagnon, J. P. (2014). The Crisis of Democracy. Which Crisis? Which Democracy? Democratic Theory, 1(2), pp. 1-10.

Foa, R. S. y Mounk, Y. (2016). The Democratic Disconnect. Journal of Democracy, 27(3), pp. 517.

Hadenius, A. y Teorell, J. (2005). Cultural and Economic Prerequisites of Democracy: Reassessing Recent Evidence. Studies in Comparative International Development, 39(4), pp. 87-106.

Held, D. (2006). Models of Democracy. Cambridge: Polity Press.

Hochschild, A. R. (2016). Strangers in their Own Land. New York: The New Press.

Hofstede, G. (1980). Culture's consequences. Beverly Hills: Sage.

Hoover, K. y Perez, S. (2007). Truth and Robustness in Cross-country Growth Regressions. Oxford Bulletin of Economics and Statistics, 66(5), pp. 765-98.

Inglehart, R. y Norris, P. (2017). Trump and the Populist Authoritarian Parties. Perspectives on Politics, 15(2), pp. 443-54.

Inglehart, R. y Norris, P. (2016). Trump, Brexit, and the rise of Populism. Paper del encuentro anual de American Political Science Association, Philadelphia.

Inglehart, R. y Welzel, C. (2005). Modernization, Cultural Change, and Democracy. Cambridge: Cambridge University Press.

Inglehart, R. (2016a). How Much Should We Worry? Journal of Democracy, 27(3), pp. 18-23.

Inglehart, R. (2016b). Inequality and Modernization. En Foreign Affairs, 95(1), pp. 2-10.

Inglehart, R. (1997). Modernization and Postmodernization. Princeton: Princeton University Press.

Inglehart, R. (1990). Culture Shift in Advanced Industrial Society. Princeton: Princeton University Press.

Jorge, J.E. (2017). Estado de Derecho y Valores Democráticos. Las Direcciones del Cambio Cultural. Question, 1(54), pp. 172-206.

Jorge, J.E. (2016a). La Confianza Interpersonal Revela sus Misterios. ¿La Partícula de Dios? Question, 1(52), pp. 143-74. 
Jorge, J.E. (2016b). Teoría de la Cultura Política. Enfocando el Caso Argentino. Question, 1(49), pp. 300-21.

Jorge, J.E. (2015). La Cultura Política Argentina: una Radiografía. Question, 1(48), pp. 372403.

Jorge, J.E. (2012). Same-sex marriage in Argentina. Tolerance and Discrimination in Political Culture. Journal of Research in Peace, Gender \& Development, 2(3), pp. 60-71.

Jorge, J.E. (2010). Cultura Política y Democracia en Argentina. La Plata: Edulp.

Kaufmann, D.; Kraay, A. y Mastruzzi, M. (2010). The Worldwide Governance Indicators. Methodology and Analytical Issues. Policy Research WP 5430, World Bank.

Kaufmann, D. y Kraay, A. (2002). Growth without Governance. Economía, 3(1), pp. 169-229.

La Porta, L.; López De-Silanes, F.; Schleifer, A. y Vishny, R. (1999). The Quality of Government. The Journal of Law, Economics \& Organization, 15(1), pp. 222-79.

Levitsky, S. y Way, L. (2015). The Myth of Democratic Recession" En Diamond, L. J. y Plattner, M. F. (pp. 58-76).

Levitsky, S. y Way, L. (2010). Competitive Authoritarianism. Cambridge: Cambridge University Press.

Licht, A. N.; Goldschmidt, C. y Schwartz, S. H. (2007). Culture rules: The foundations of the rule of law and other norms of governance. En Journal of Comparative Economics, 35, pp. 659-68.

Luce, E.E. (2017). The Retreat of Western Liberalism. New York: Atlantic Monthly Press.

Merkel, W. (2016). Democracies and Their Crises Reconsidered. Democratic Theory, 3(1), pp. 91-108.

Merkel, W. (2004). Embedded and Defective Democracies. Democratization, 11(5), pp. 33-58.

Merkel, W. y Bochsler, D. et al. (2016). Democracy Barometer. Methodology. Version 5. Aarau: Zentrum für Demokratie.

Milanovic, B. (2016). Global Inequality. Cambridge: The Belknap Press of Harvard University Press.

Munck, G. L. (2009). Measuring Democracy. Baltimore: Johns Hopkins University Press.

Pemstein, D.; Meserve, S. A. y Melton, J. (2010). Democratic Compromise: A Latent Variable Analysis of Ten Measures of Regime Type. Political Analysis, 18(4), pp. 426-49.

Piketty, T. (2014). Capital in the Twenty-First Century. Cambridge: The Belknap Press of Harvard University Press.

Price, G. N. (2003). Economic Growth in a Cross-section of Nonindustrial Countries. En Review of Development Economics, 7(3), pp. 478-95.

Rothman, J. (2016). How to restore your faith in democracy. The New Yorker, 11 de noviembre.

Schwartz, S. H. (2009). Culture Matters. En Wyer, R. S.; Chiu, C. Y. y Hong, Y. Y. (eds.). Understanding Culture (pp. 127-50). New York: Psychology Press. 
Schwartz, S.H. (2008a). National Cultural Value Orientations Scores for 80 Countries. Research Gate. Recuperado de https://doi.org/10.13140/RG.2.1.3313.3040

Schwartz, S. H. (2008b). Cultural value orientations: Nature and implications of national differences. Moscow: State University, Higher School of Economics Press.

Schwartz, S. H. (2006). A Theory of Cultural Value Orientations. Explication and Applications. En Comparative Sociology, 5(2-3), pp. 136-182.

Schwartz, S. H. (1992). Universals in the content and structure of values. En Zanna, M. (ed.). Advances in Experimental Social Psychology (pp. 1-65). New York: Academic Press.

Treier, S. y Jackman, S. (2008). Democracy as a latent variable. American Journal of Political Science, 52(1), pp. 201-17.

Welzel, C. y Kirsch, H. (2017). Democracy Misunderstood: Authoritarian Notions of Democracy around the Globe. World Values Research, 9(1), pp. 1-29.

Welzel, C.; Inglehart, R. y Kruse, S. (2016). Pitfalls in the Study of Democratization. Bristish Journal of Political Science, 47(2), pp. 463-72.

Welzel, C. (2013). Freedom Rising. New York: Cambridge University Press.

Welzel, C. (2010). How Selfish Are Self-Expression Values? A Civicness Test. Journal of CrossCultural Psychology, 41, pp. 152-74. 
Anexo

Tabla: Muestra de paises y principales datos

Período WVS 2003-2014 Período SVS 1990s-2007

\begin{tabular}{|c|c|c|c|c|c|c|c|c|c|c|c|c|c|c|c|c|c|}
\hline \multirow{3}{*}{ aís } & & \\
\hline & ICPD & EDI & EDI & $\mathrm{FH}$ & & & & BS & \multirow{2}{*}{\multicolumn{9}{|c|}{$\begin{array}{l}\text { Aut Igu ICPD EDI EDI } \\
\text { Inm Jer Base I II FH VAI DB BTI }\end{array}$}} \\
\hline & Base & I & II & $\mathbf{S}$ & & & & G & & & & & & & & & \\
\hline Albania & 18 & & & & 5,3 & & & & & & & & & & & & \\
\hline Alemania & 50 & 8,4 & 7,8 & 9,6 & 9,0 & & 6,3 & 8,6 & 1,5 & 3,2 & 55 & 8,4 & 8,1 & 9,6 & 8,9 & 6,2 & \\
\hline Argelia & 15 & 1,3 & 1,3 & 3,5 & 3,4 & 3,9 & & & & & & & & & & & \\
\hline Argentina & 37 & 2,9 & 5,7 & 8,0 & 6,2 & 7,3 & 4,8 & & 0,5 & 2,9 & 37 & 3,2 & 5,7 & 7,8 & 6,3 & 4,8 & 7,6 \\
\hline Armenia & 12 & 1,5 & 1,5 & 3,9 & 3,9 & 4,5 & & & & & & & & & & & \\
\hline Australia & 55 & 8,6 & 8,9 & 9,6 & 9,2 & & 6,0 & 7,5 & 0,5 & 2,5 & 57 & 8,9 & 9,0 & 10 & 9,1 & 6,3 & \\
\hline Austria & & & & & & & & 7,2 & 1,5 & 3,1 & 39 & 9,1 & 8,2 & 10 & 8,9 & 6,0 & \\
\hline Azerbaiján & 11 & 0,8 & 0,8 & 2,7 & 2,5 & 3,3 & & & & & & & & & & & \\
\hline Bangladesh & 16 & & & & 4,4 & & & & & & & & & & & & \\
\hline Bélgica & & & & & & & & 7,1 & 1,0 & 3,5 & 44 & 7,5 & 8,9 & 9,6 & 9,0 & 6,6 & \\
\hline Bielorrusia & 23 & 0,5 & 0,4 & 1,5 & 1,7 & 3,3 & & & & & & & & & & & \\
\hline Bolivia & & & & & & & & & $-0,6$ & 2,1 & & 2,8 & 5,7 & 7,5 & 5,6 & 4,6 & 6,2 \\
\hline Bosnia & 25 & & & & 5,0 & & & & $-0,3$ & 2,9 & 23 & 1,9 & 3,1 & 4,7 & 5,5 & & 6,4 \\
\hline Brasil & 40 & 3,7 & 5,0 & 8,1 & 6,5 & 7,9 & 5,0 & & 0,3 & 2,5 & 38 & 3,2 & 4,7 & 7,1 & 6,4 & 5,0 & 7,7 \\
\hline Bulgaria & 26 & 4,1 & 6,5 & 8,7 & 7,0 & 8,3 & 5,1 & 5,3 & 0,0 & 1,5 & 23 & 3,8 & 5,5 & 8,4 & 6,8 & 4,9 & 8,4 \\
\hline B. Faso & 19 & 2,3 & 3,6 & 5,3 & 4,7 & 5,3 & & & & & & & & & & & \\
\hline Camerún & & & & & & & & & $-1,6$ & 2,2 & & 0,4 & 0,6 & 1,5 & 3,0 & & 3,5 \\
\hline Canadá & 58 & 8,8 & 8,1 & 9,8 & 9,1 & & 6,2 & 7,4 & 1,0 & 2,8 & 58 & 9,0 & 9,0 & 10 & 9,3 & 6,3 & \\
\hline Chile & 37 & 7,8 & 8,0 & 9,7 & 8,2 & 9,2 & 5,2 & 6,2 & 0,0 & 2,8 & 31 & 7,1 & 7,0 & 9,0 & 7,9 & 5,1 & 9,1 \\
\hline China & 21 & 0,7 & 0,0 & 1,7 & 1,7 & 2,6 & & & 0,0 & 0,7 & 23 & 0,3 & 0,1 & 0,7 & 2,0 & & 2,3 \\
\hline Chipre & 28 & 6,8 & 7,9 & 9,4 & 8,1 & & 5,5 & 5,8 . & $-0,5$ & 2,9 & 30 & 7,3 & 8,2 & 10 & 8,1 & 5,3 & \\
\hline Colombia & 32 & 2,6 & 2,5 & 6,1 & 5,3 & 5,8 & 4,8 & & 0,1 & 1,8 & 29 & 2,2 & 2,1 & 5,5 & 4,6 & 4,5 & 5,3 \\
\hline Corea del & 25 & 5,6 & 6,0 & 8,6 & 7,3 & 8,7 & & 5,1 & 0,2 & 1,5 & 27 & 5,4 & 5,9 & 8,6 & 7,1 & & 8,8 \\
\hline Costa Rica & & & & & & & & & 0,4 & 2,6 & & 5,8 & 8,3 & 9,4 & 8,0 & 4,7 & 9,4 \\
\hline Croacia & & & & & & & & 5,4 & 0,1 & 2,1 & 36 & 3,4 & 5,4 & 7,2 & 6,4 & 4,7 & 8,9 \\
\hline Dinamarca & & & & & & & & 8,8 & 1,4 & 3,2 & 54 & 9,6 & 9,8 & 10 & 9,5 & 7,3 & \\
\hline Ecuador & 27 & 1,9 & 3,7 & 6,1 & 4,9 & 5,2 & 4,5 & & & & & & & & & & \\
\hline EEUU & 48 & 7,6 & 7,4 & 9,4 & 8,3 & & 5,4 & 7,9 & 0,4 & 2,3 & 51 & 8,3 & 7,9 & 10 & 8,7 & 5,9 & \\
\hline Egipto & 10 & 1,3 & 0,7 & 3,5 & 3,2 & 3,9 & & & $-1,3$ & 2,2 & 13 & 0,9 & 0,7 & 2,1 & 3,2 & & 3,6 \\
\hline Eslovaquia & 29 & & & & 7,3 & & & 6,7 . & $-0,2$ & 2,6 & 28 & 5,1 & 7,7 & 9,0 & 7,7 & 5,3 & 9,1 \\
\hline Eslovenia & 43 & 6,3 & 8,7 & 9,1 & 8,1 & 9,7 & 6,0 & 7,3 & 0,6 & 2,9 & 39 & 6,8 & 8,8 & 9,6 & 8,3 & 6,0 & 9,6 \\
\hline España & 42 & 7,1 & 6,8 & 9,7 & 8,2 & & 5,4 & 6,5 & 1,0 & 3,4 & 41 & 7,3 & 6,9 & 9,6 & 8,5 & 5,6 & \\
\hline Estonia & 32 & 6,8 & 8,6 & 9,5 & 8,3 & 9,5 & 5,2 & 8,1 & 0,0 & 2,5 & 26 & 6,2 & 8,6 & 9,4 & 8,0 & 5,2 & 9,4 \\
\hline Etiopia & 33 & 1,3 & 0,9 & 3,4 & 2,6 & 3,5 & & & $-1,3$ & 2,1 & 33 & 1,2 & 1,3 & 3,6 & 2,8 & & 3,5 \\
\hline $\mathrm{Fiji}$ & & & & & & & & & $-0,8$ & 2,1 & & 2,6 & 4,3 & 5,4 & 5,3 & & \\
\hline Filipi & 21 & 2,4 & 2,5 & 6,2 & 5,5 & 6,0 & & & $-0,6$ & 1,9 & 18 & 3,0 & 3,8 & 7,2 & 5,8 & & 6,3 \\
\hline Finlandia & 58 & 9,7 & 9,5 & 9,9 & 9,7 & & 7,0 & 9,0 & 1,1 & 3,1 & 55 & 9,7 & 9,7 & 10 & 9,5 & 7,0 & \\
\hline Francia & 54 & 7,5 & 8,1 & 9,4 & 8,8 & & 5,1 & 6,7 & 1,6 & 2,8 & 48 & 7,6 & 8,3 & 9,6 & 8,6 & 5,2 & \\
\hline Georgia & 14 & 2,5 & 3,4 & 5,6 & 4,9 & 5,9 & & & $-0,4$ & 2,2 & 14 & 1,8 & 2,9 & 5,6 & 4,9 & & 6,1 \\
\hline Ghana & 12 & 4,2 & 5,4 & 8,4 & 6,6 & 8,0 & & & $-1,1$ & 2,1 & 14 & 3,6 & 5,4 & 7,7 & 5,7 & & 7,8 \\
\hline G. Bretaña & 55 & 8,4 & 8,3 & 9,7 & 8,9 & & 5,7 & 7,0 & 1,1 & 2,6 & 52 & 8,5 & 8,2 & 9,6 & 8,9 & 5,6 & \\
\hline Grecia & & & & & & & & 6,6 & 0,7 & 3,0 & & 5,6 & 6,3 & 8,8 & 8,0 & 5,2 & \\
\hline Guatemala & 29 & 1,7 & 3,9 & 5,3 & 4,9 & 5,2 & 3,8 & & & & & & & & & & \\
\hline Hungría & 25 & & & & 8,2 & & & 3,8 & 0,5 & 2,6 & 26 & 6,2 & 8,3 & 9,4 & 8,2 & 5,6 & 9,3 \\
\hline India & 23 & 3,4 & 1,9 & 7,7 & 6,5 & 7,8 & 3,7 & & $-0,2$ & 1,4 & 22 & 3,5 & 2,4 & 7,4 & 6,5 & 4,6 & 7,8 \\
\hline Indonesia & 18 & 2,2 & 2,7 & 6,4 & 5,3 & 5,9 & & & $-0,6$ & 1,8 & 18 & 1,7 & 1,3 & 5,3 & 4,6 & & 6,0 \\
\hline Irak & 14 & 0,5 & 0,7 & 2,5 & 2,8 & 3,6 & & & & & & & & & & & \\
\hline Irán & 15 & 0,8 & 0,1 & 2,4 & 1,9 & 3,1 & & & $-0,7$ & 1,3 & 20 & 0,6 & 0,2 & 1,5 & 2,7 & & 3,1 \\
\hline Irlanda & & & & & & & & 8,1 & 0,9 & 2,8 & 41 & 8,3 & 9,3 & 10 & 9,0 & 5,8 & \\
\hline Israel & & & & & & & & 6,5 & 0,4 & 2,2 & 31 & 6,2 & 3,9 & 8,6 & 7,1 & 5,3 & \\
\hline
\end{tabular}


Tabla (continuación)

\begin{tabular}{|c|c|c|c|c|c|c|c|c|c|c|c|c|c|c|c|c|c|}
\hline \multirow[b]{2}{*}{ País } & \multicolumn{8}{|c|}{ Período WVS 2003-2014 } & \multicolumn{9}{|c|}{ Período SVS 1990s-2007 } \\
\hline & $\begin{array}{l}\text { ICPD } \\
\text { Base }\end{array}$ & $\begin{array}{c}\text { EDI } \\
\text { I }\end{array}$ & $\begin{array}{c}\text { EDI } \\
\text { II }\end{array}$ & $\begin{array}{c}\mathrm{FH} \\
\mathrm{S}\end{array}$ & VAI & BTI & DB & $\begin{array}{c}\text { BS } \\
\text { G }\end{array}$ & $\begin{array}{l}\text { Aut } \\
\text { Inm }\end{array}$ & $\begin{array}{l}\text { Igu } \\
\text { Jer }\end{array}$ & $\begin{array}{l}\text { ICPD } \\
\text { Base }\end{array}$ & $\begin{array}{c}\text { EDI } \\
\text { I }\end{array}$ & $\begin{array}{c}\text { EDI } \\
\text { II }\end{array}$ & FH & VAl & DB & BTI \\
\hline Italia & 44 & 5,4 & 7,6 & 9,2 & 8,2 & & 5,7 & 6,8 & 0,7 & 3,7 & 41 & 5,9 & 8,4 & 9,6 & 8,1 & 5,8 & \\
\hline Japón & 38 & 6,8 & 8,5 & 8,8 & 8,0 & & 5,4 & 5,5 & 0,8 & 1,7 & 38 & 6,9 & 8,6 & 9,2 & 7,9 & 5,3 & \\
\hline Jordania & 9 & 2,0 & 1,0 & 3,5 & 3,6 & 3,4 & & & $-0,5$ & 1,9 & 11 & 2,3 & 1,8 & 4,2 & 4,3 & & 3,4 \\
\hline Kazajistán & 18 & 0,9 & 0,9 & 2,8 & 2,8 & 3,4 & & & & & & & & & & & \\
\hline Kirguistán & 16 & 1,0 & 1,5 & 3,8 & 3,7 & 4,3 & & & & & & & & & & & \\
\hline Letonia & & & & & & & & 7,8 & 0,0 & 2,5 & 31 & 5,0 & 7,7 & 9,2 & 7,4 & 5,0 & 8,3 \\
\hline Líbano & 19 & 1,6 & 2,2 & 4,9 & 4,5 & 5,6 & & & & & & & & & & & \\
\hline Libia & 11 & 0,6 & 0,5 & 3,6 & 2,7 & 2,5 & & & & & & & & & & & \\
\hline Macedonia & 22 & & & & 4,8 & & & & $-0,3$ & 1,7 & 20 & 2,4 & 4,9 & 6,3 & 5,2 & & 7,4 \\
\hline Malasia & 12 & 2,8 & 1,7 & 4,9 & 4,7 & 4,6 & & & $-0,8$ & 2,2 & 14 & 2,4 & 1,8 & 4,1 & 4,7 & & 4,7 \\
\hline Malí & 18 & 3,2 & 5,1 & 7,3 & 6,1 & 7,0 & & & & & & & & & & & \\
\hline Marruecos & 12 & 1,9 & 1,7 & 4,2 & 3,9 & 3,3 & & & & & & & & & & & \\
\hline México & 37 & 2,6 & 3,1 & 6,6 & 5,8 & 6,7 & 4,6 & 5,4 & $-0,3$ & 2,6 & 35 & 3,0 & 3,8 & 7,2 & 5,9 & 4,6 & 7,2 \\
\hline Moldavia & 21 & 2,2 & 3,4 & 5,7 & 4,7 & 4,9 & 4,2 & & & & & & & & & & \\
\hline Namibia & & & & & & & & & $-0,4$ & 2,0 & & 4,2 & 5,8 & 7,7 & 6,4 & & 7,7 \\
\hline Nepal & & & & & & & & & $-0,7$ & 1,6 & & 1,9 & 1,7 & 4,9 & 3,9 & & 3,1 \\
\hline Nigeria & 15 & 1,2 & 0,4 & 4,7 & 3,7 & 4,2 & & & $-1,3$ & 2,1 & 15 & 1,0 & 1,1 & 4,1 & 3,4 & & 5,6 \\
\hline Noruega & 70 & 9,1 & 9,4 & 10 & 9,4 & & 6,7 & 8,9 & 0,7 & 3,6 & 65 & 9,3 & 9,8 & 10 & 9,4 & 6,8 & \\
\hline N. Zelanda & 55 & 9,2 & 9,4 & 9,7 & 9,5 & & 6,3 & 8,4 & 1,2 & 2,7 & 59 & 9,4 & 9,7 & 10 & 9,5 & 6,4 & \\
\hline Omán & & & & & & & & & $-1,2$ & 2,3 & & 1,3 & 1,2 & 2,2 & 3,6 & & 2,9 \\
\hline Países & 54 & 9,1 & 9,2 & 9,9 & 9,5 & & 6,7 & 7,4 & 1,3 & 3,1 & 56 & 9,1 & 9,9 & 10 & 9,5 & 6,7 & \\
\hline Pakistán & 20 & 1,3 & 0,5 & 4,3 & 3,5 & 2,8 & & & $-0,9$ & 2,2 & 13 & 0,9 & 0,7 & 2,9 & 3,1 & & 2,9 \\
\hline Perú & 29 & 2,8 & 4,5 & 7,1 & 5,7 & 6,4 & 4,1 & & $-0,3$ & 2,1 & 31 & 2,6 & 3,9 & 6,5 & 5,2 & 4,1 & 6,4 \\
\hline Polonia & 34 & 5,9 & 7,8 & 9,3 & 8,0 & 9,1 & 5,5 & 8,2 & $-0,1$ & 2,0 & 26 & 5,6 & 8,1 & 9,4 & 7,9 & 5,6 & 8,9 \\
\hline Portugal & & & & & & & & 7,1 & 0,7 & 3,3 & 32 & 7,5 & 9,3 & 10 & 8,9 & 5,8 & \\
\hline R. Checa & 36 & & & & 7,7 & & & 7,1 & 0,5 & 2,2 & 33 & 5,9 & 8,1 & 9,4 & 7,8 & 5,3 & 9,4 \\
\hline Ruanda & 18 & 1,4 & 0,8 & 2,5 & 2,5 & 3,3 & & & & & & & & & & & \\
\hline Rumania & 20 & 4,0 & 5,0 & 8,2 & 6,3 & 8,1 & 4,4 & 4,6 & 0,3 & 2,5 & 20 & 3,7 & 5,6 & 8,2 & 6,5 & 4,4 & 8,2 \\
\hline Rusia & 22 & 0,8 & 0,3 & 2,7 & 3,4 & 4,8 & & & 0,1 & 1,7 & 19 & 1,1 & 1,2 & 3,7 & 4,1 & & 5,0 \\
\hline Senegal & & & & & & & & & $-1,3$ & 2,3 & & 3,0 & 3,9 & 6,4 & 5,7 & & 7,1 \\
\hline Serbia & 30 & 3,0 & 5,3 & 7,6 & 5,8 & 7,1 & & & 0,6 & 2,8 & 30 & 2,5 & 3,3 & 7,5 & 4,7 & & 7,3 \\
\hline Singapur & 23 & 4,6 & 3,5 & 5,1 & 5,6 & 4,9 & & & $-0,4$ & 1,8 & 21 & 3,4 & 2,3 & 3,8 & 5,5 & & 4,8 \\
\hline Sudáfrica & 26 & 4,1 & 4,7 & 8,1 & 7,0 & 7,4 & 5,1 & & $-0,4$ & 1,9 & 24 & 5,1 & 6,2 & 9,0 & 7,3 & 5,3 & 8,5 \\
\hline Suecia & 69 & 9,5 & 8,7 & 10 & 9,6 & & 7,0 & 9,1 & 1,6 & 3,1 & 69 & 9,3 & 9,4 & 10 & 9,4 & 6,9 & \\
\hline Suiza & 63 & 9,1 & 8,3 & 9,8 & 9,4 & & 6,9 & 8,5 & 1,3 & 2,6 & 57 & 9,3 & 9,1 & 10 & 9,2 & 6,8 & \\
\hline $\mathrm{T}$ Tobago & 27 & 3,6 & 6,5 & 8,1 & 6,7 & & & & & & & & & & & & \\
\hline Tailandia & 23 & 2,4 & 1,7 & 5,3 & 4,3 & 4,3 & 4,2 & & $-0,2$ & 1,1 & 21 & 3,3 & 3,8 & 6,5 & 5,7 & 4,4 & 5,4 \\
\hline Taiwán & 27 & 6,1 & 7,8 & 8,8 & 7,7 & 9,6 & & & 0,0 & 1,6 & 19 & 5,8 & 7,6 & 8,8 & 7,5 & & 9,3 \\
\hline Tanzania & 20 & & & & 4,4 & & & & & & & & & & & & \\
\hline Túnez & 10 & 2,8 & 1,8 & 6,0 & 5,4 & 4,3 & & & & & & & & & & & \\
\hline Turquía & 15 & 3,3 & 2,4 & 6,3 & 5,2 & 7,4 & 3,6 & 3,9 & 0,1 & 1,8 & 19 & 2,5 & 1,8 & 5,3 & 5,0 & 3,9 & 6,7 \\
\hline Ucrania & 23 & 2,1 & 3,9 & 6,7 & 5,1 & 6,2 & 4,3 & & $-0,2$ & 1,8 & 20 & 1,9 & 3,3 & 6,0 & 4,7 & 4,3 & 6,9 \\
\hline Uganda & 19 & & & & 3,1 & & & & $-1,0$ & 1,4 & 19 & 1,4 & 1,5 & 4,0 & 3,8 & & 5,6 \\
\hline Uruguay & 42 & 6,8 & 8,4 & 9,7 & 8,2 & 9,9 & 5,1 & & & & & & & & & & \\
\hline Uzbekistán & 13 & 0,1 & 0,1 & 0,4 & 0,6 & 2,1 & & & & & & & & & & & \\
\hline Venezuela & 28 & & & & 5,0 & & & & 0,1 & 2,7 & 29 & 1,6 & 2,9 & 5,8 & 4,4 & 3,9 & 4,5 \\
\hline Vietnam & 21 & 0,7 & 0,5 & 1,9 & 2,0 & 2,3 & & & & & & & & & & & \\
\hline Yemen & 12 & 0,6 & 0,1 & 2,5 & 2,2 & 2,5 & & & $-1,6$ & 2,5 & 12 & 0,8 & 0,9 & 2,8 & 3,2 & & 3,3 \\
\hline Zambia & 25 & 2,2 & 3,3 & 5,8 & 5,1 & 6,3 & & & & & & & & & & & \\
\hline Zimbabwe & 21 & 0,4 & 0,2 & 2,2 & 2,0 & 3,6 & & & $-0,3$ & 1,6 & 18 & 0,5 & 0,7 & 1,9 & 2,4 & & 3,3 \\
\hline
\end{tabular}


Notas de la Tabla. Aut-Inm: Autonomía menos Inmersión. Igu-Jer: Igualitarismo menos Jerarquía. Nota técnica. Los puntajes por país de los índices de democracia del periodo WVS son promedios trianuales (bianuales para BTI) centrados en el año en que WVS relevó los datos del índice ICPD. En casos puntuales se usan los datos disponibles más cercanos al año WVS. Los promedios de BSG son del periodo 2014-16, único disponible del nuevo índice anual. El año WVS por país se encuentra en Jorge (2015). Los pùntajes de los índices de democracia del periodo SVS son promedios de todo el periodo 1996-2007 (criterio empleado por Licht, Goldschmidt \& Schwartz, 2007). Los puntajes de los valores culturales de Schwartz son de Schwartz (2008a). En los casos de Canadá, Suiza e Israel, se calcularon puntajes únicos por país ponderando los de los subgrupos étnicos o lingüisticos según su peso en los índices de Alesina et al., 2003. Los puntajes de las dos regiones de Alemania se ponderaron según su peso poblacional. Los datos del ICPD Base del periodo SVS son del lapso 1990-2007. Para los paises con datos del ICPD en más de un año de este periodo, se calculó el promedio de todos los puntos temporales disponibles, 